\title{
An analytical approach to the unified solution of kinetic equations in rarefied gas dynamics. I. Flow problems
}

\author{
C. S. Scherer, J. F. Prolo Filho and L. B. Barichello*
}

\begin{abstract}
The ADO method, an analytical version of the discrete-ordinates method, is used to solve several classical problems in the rarefied gas dynamics field. The complete development of the solution, which is analytical in terms of the spatial variable, is presented in a way, such that, a wide class of kinetic models are considered, in an unified approach. A series of numerical results are showed and different simulations are used in order to establish a general comparative analysis based on this consistent set of results provided by the same methodology.
\end{abstract}

Keywords. Rarefied gas dynamics, kinetic models, ADO method, discrete ordinates.

\section{Introduction}

It is well known that under certain conditions, the behavior of a gas flow is described by the solution of the Boltzmann equation [1]. Although this is the case for important classical applications, such as vacuum equipments [2] and aerodynamic applications [3], the interest in new technological applications, particularly associated with micro-systems [4], has brought more attention, in these days, to the use of approaches based on the Boltzmann equation [5]. In fact, even in the continuum regime, procedures like the Lattice-Boltzmann method [6], has been based on the theory originally proposed by Boltzmann and related to the kinetic theory of gases.

In this context, due to the complexity of the original mathematical model and the consequent computational and numerical difficulties associated, the so-called kinetic models (kinetic equations) have played a very important role in providing simpler mathematical models associated with the Boltzmann formulation, which keep physical features of the original one and that are more amenable to the development of analytical and numerical tools, in order to obtain numerical results for physical quantities of interest. Some of these kinetic models have been widely studied and used over the years, like the well known BGK model [7]. Extensions

* Corresponding author 
and improvements have been proposed to that formulation, like the Gross-Jackson (GJ) model [8], the S-model [9] and, more recently, the CES [10], the CEBS [10] and the MRS [11] models. In particular, recent works $[11,12,13]$ note that results for the S-model formulation, as well as the GJ and MRS models, can be obtained as particular cases (one gas) of the McCormack model [14] for mixtures. In deriving all of the listed models, the collision frequency of the particles is considered not dependent of the velocity. However, velocity dependent models were also proposed, like the CLF model $[15,16]$. Results based on all these models, for specific problems, were obtained by different authors, over the years [17]-[24], most of them based on numerical approaches.

Following the recent interest in rarefied gas dynamics (RGD) applications, in the past few years, a methodology initially proposed for radiative transfer problems, the ADO method [25], has been used in connection with some problems in that field. In particular, a series of studies related to the derivation [10] and solution of model equations has been developed. In summary, the same approach has been used to solve in an "unified" manner many classical problems in RGD, based on specific model equations: like the BGK model [26], the S-model [27], the CLF model [28] and the CES model [29, 30]. In general, the ADO solution has been shown to be concise, accurate and easy to implement. However, it is possible to note, as could be expected of an analytical procedure, that, in developing the solution, some steps may arise as special topics for each one of the kinetic equations and the specific problems to be solved. That is the case, particularly, for two important issues which arise in the ADO solution - the derivation of an eigenvalue problem and the derivation of exact solutions associated with the presence of degenerate eigenvalues.

In this work, we pursue the goal of having a solution as much general as possible, which we believe is an important aspect when it comes to analytical approaches. Then, following a general procedure, we develop the complete ADO solution, valid for different kinetic equations, all of them described in a same general expression, and for a wide class of problems. In this way, based on our solution, we present a collection of results obtained by the ADO method for many classical flow problems in the rarefied gas dynamics, which comprises previous developed cases, but where we also include original results for the Gross--Jackson (GJ) and the MRS models, and, we still add, for all models, the case of channels defined by different surfaces (different accommodation coefficients). In this sense, a comparative analysis with results from different models, provided by the same methodology, can be carried out.

This work is organized such that, we present in the next section the general formulation of all the problems we deal with. In Sec. 3 we present the transformation of the scalar problem in an appropriate vector form, in order to apply the ADO method. In Secs. 4 and 5 the ADO solution is developed for flow problems. The evaluation of the physical quantities of interest is presented in Sec. 6. Finally, Secs. 7 and 8 are devoted to the discussion of computational aspects, numerical 
results and the indication of some concluding remarks.

\section{Kinetic equations}

We choose to start this work with the linearized Boltzmann equation (LBE), written, in the form $[21,10]$

$c_{x} \frac{\partial}{\partial \tau} h(\tau, \mathbf{c})+\varepsilon h(\tau, \mathbf{c})=\varepsilon \pi^{-3 / 2} \int_{-\infty}^{\infty} \int_{-\infty}^{\infty} \int_{-\infty}^{\infty} \mathrm{e}^{-c^{\prime 2}} h\left(\tau, \mathbf{c}^{\prime}\right) F\left(\mathbf{c}^{\prime}: \mathbf{c}\right) \mathrm{d} c_{x}^{\prime} \mathrm{d} c_{y}^{\prime} \mathrm{d} c_{z}^{\prime}+S(\mathbf{c})$,

with an inhomogeneous source term $S(\mathbf{c})$,

$$
\mathbf{c}=\mathbf{v}\left(m_{0} / 2 k T_{0}\right)^{1 / 2}
$$

where $k$ is the Boltzmann constant, $T_{0}$ is a reference temperature and $m_{0}$ is the mass of a gas particle. In addition to the three components of the velocity vector $\left(c_{x}, c_{y}, c_{z}\right)$, which are expressed in dimensionless units, we consider here the dimensionless (written in terms of a mean-free path $l$ ) spatial variable $\tau$, and

$$
\varepsilon=\sigma_{0}^{2} n_{0} \pi^{1 / 2} l,
$$

where $\sigma_{0}$ is the collision diameter of the gas particles (in the rigid-sphere approximation) and $n_{0}$ is the equilibrium density of the gas. For rigid-spheres, the exact scattering kernel $F\left(\mathbf{c}^{\prime}: \mathbf{c}\right)$ can be expanded in terms of Legendre functions [31]. As showed in previous work [10], in order to obtain simpler mathematical and numerical formulations, truncated forms of the expansion proposed by Pekeris and Alterman [31] can be used to define, by imposing appropriate conditions, a class of model equations. Particularly, in regard to the kinetic models discussed in this work, two of them (BGK and S) are defined by two terms in that truncated expansion, while the GJ and the MRS models are defined by three terms. In fact, for appropriate choices of the parameters $\beta$ and $\varpi$, following previous references $[32,33,34,11]$, we can represent all of the kinetic equations we want to discuss here, in a general expression

$$
F\left(\mathbf{c}^{\prime}: \mathbf{c}\right)=1+2\left(\mathbf{c}^{\prime} \cdot \mathbf{c}\right)+(2 / 3)\left(c^{\prime 2}-3 / 2\right)\left(c^{2}-3 / 2\right)+\beta M\left(\mathbf{c}^{\prime}: \mathbf{c}\right)+\varpi N\left(\mathbf{c}^{\prime}: \mathbf{c}\right),
$$

with

$$
M\left(\mathbf{c}^{\prime}: \mathbf{c}\right)=(4 / 5)\left(\mathbf{c}^{\prime} \cdot \mathbf{c}\right)\left(c^{\prime 2}-5 / 2\right)\left(c^{2}-5 / 2\right)
$$

and

$$
N\left(\mathbf{c}^{\prime}: \mathbf{c}\right)=2\left[\left(\mathbf{c}^{\prime} \cdot \mathbf{c}\right)^{2}-(1 / 3) c^{\prime 2} c^{2}\right] .
$$

Furthermore, as showed by Barichello and Siewert [10], depending on the kinetic model, $\varepsilon$ also assumes different values, when defined in terms of a mean-free-path evaluated in terms of viscosity $\left(\varepsilon_{p}\right)$ or thermal-conductivity $\left(\varepsilon_{t}\right)$.

To be clear, we complete the definition of Eqs. (1) and (4) as follows [32, 10, $11,34]$, 
- the BGK model

$$
\beta=\varpi=0, \quad \varepsilon_{t}=\varepsilon_{p}=1, \quad \varepsilon_{p} / \varepsilon_{t}=1
$$

- the $\mathrm{S}$ model

$$
\beta=1 / 3, \quad \varpi=0, \quad \varepsilon_{t}=3 / 2, \quad \varepsilon_{p}=1, \quad \varepsilon_{p} / \varepsilon_{t}=2 / 3
$$

- the Gross-Jackson (GJ) model

$$
\beta=5 / 9, \quad \varpi=1 / 3, \quad \varepsilon_{t}=9 / 4, \quad \varepsilon_{p}=3 / 2, \quad \varepsilon_{p} / \varepsilon_{t}=2 / 3
$$

- the MRS model

$$
\begin{array}{r}
\beta=1-(16 / 15) 2^{1 / 2}, \quad \varpi=1-(8 / 5) 2^{1 / 2}, \quad \varepsilon_{t}=(15 / 32) 2^{1 / 2}, \\
\varepsilon_{p}=(5 / 16) 2^{1 / 2}, \quad \varepsilon_{p} / \varepsilon_{t}=2 / 3 .
\end{array}
$$

As pointed out previously [10], the ratio $\varepsilon_{p} / \varepsilon_{t}$, assumed as an expected value for the Prandtl number, may be an aspect which establishes a difference between the BGK model and the other ones, although all of them are constant collision frequency models.

Once we have the basic kinetic equation defined, to complete the definition of an specific problem to be solved, we supplement Eq. (1) with boundary conditions. Here we consider specular and diffuse reflection, such that, for plane channel problems, $\tau \in[-a, a]$, we write them, for $c_{x}>0$, as

$$
\begin{aligned}
h\left(-a, c_{x}, c_{y}, c_{z}\right)- & \left(1-\alpha_{1}\right) h\left(-a,-c_{x}, c_{y}, c_{z}\right) \\
& =\frac{2 \alpha_{1}}{\pi} \int_{-\infty}^{\infty} \int_{-\infty}^{\infty} \int_{0}^{\infty} \mathrm{e}^{-c^{\prime 2}} h\left(-a,-c_{x}^{\prime}, c_{y}^{\prime}, c_{z}^{\prime}\right) c_{x}^{\prime} \mathrm{d} c_{x}^{\prime} \mathrm{d} c_{y}^{\prime} \mathrm{d} c_{z}^{\prime}
\end{aligned}
$$

and

$$
\begin{aligned}
h\left(a,-c_{x}, c_{y}, c_{z}\right)- & \left(1-\alpha_{2}\right) h\left(a, c_{x}, c_{y}, c_{z}\right) \\
= & \frac{2 \alpha_{2}}{\pi} \int_{-\infty}^{\infty} \int_{-\infty}^{\infty} \int_{0}^{\infty} \mathrm{e}^{-c^{\prime 2}} h\left(a, c_{x}^{\prime}, c_{y}^{\prime}, c_{z}^{\prime}\right) c_{x}^{\prime} \mathrm{d} c_{x}^{\prime} \mathrm{d} c_{y}^{\prime} \mathrm{d} c_{z}^{\prime},
\end{aligned}
$$

where we allow different accommodation coefficients $\alpha_{i} \in(0,1]$, for $i=1,2$, to represent channels defined by different surfaces. We note that, results for the Cercignani-Lampis kernel have been also recently obtained [32, 35, 13], although we are not going to treat this case, in this work.

To complete this section we express in terms of the distribution $h$ some basic quantities of interest [32] we want to evaluate in this work, as the velocity profile

$$
u(\tau)=\pi^{-3 / 2} \int_{-\infty}^{\infty} \int_{-\infty}^{\infty} \int_{-\infty}^{\infty} \mathrm{e}^{-c^{2}} h\left(\tau, c_{x}, c_{y}, c_{z}\right) c_{y} \mathrm{~d} c_{x} \mathrm{~d} c_{y} \mathrm{~d} c_{z}
$$


the heat flow profile

$$
q(\tau)=\pi^{-3 / 2} \int_{-\infty}^{\infty} \int_{-\infty}^{\infty} \int_{-\infty}^{\infty} \mathrm{e}^{-c^{2}} h\left(\tau, c_{x}, c_{y}, c_{z}\right)\left(c^{2}-5 / 2\right) c_{y} \mathrm{~d} c_{x} \mathrm{~d} c_{y} \mathrm{~d} c_{z},
$$

the mass flow and heat flow rates, in general,

$$
U=\frac{1}{2 a^{2}} \int_{-a}^{a} u(\tau) \mathrm{d} \tau
$$

and

$$
Q=\frac{1}{2 a^{2}} \int_{-a}^{a} q(\tau) \mathrm{d} \tau
$$

\section{Basic formulation}

In order to reduce the number of variables involved in the problem, and taking into account the way the quantities of interest are defined in terms of $h$, we define

$$
\begin{gathered}
g_{1}\left(\tau, c_{x}\right)=\int_{-\infty}^{\infty} \int_{-\infty}^{\infty} \phi_{1}\left(c_{y}, c_{z}\right) h\left(\tau, c_{x}, c_{y}, c_{z}\right) \mathrm{d} c_{y} \mathrm{~d} c_{z}, \\
g_{2}\left(\tau, c_{x}\right)=\int_{-\infty}^{\infty} \int_{-\infty}^{\infty} \phi_{2}\left(c_{y}, c_{z}\right) h\left(\tau, c_{x}, c_{y}, c_{z}\right) \mathrm{d} c_{y} \mathrm{~d} c_{z}
\end{gathered}
$$

with

$$
\phi_{1}\left(c_{y}, c_{z}\right)=\left(c_{y} / \pi\right) \mathrm{e}^{-\left(c_{y}^{2}+c_{z}^{2}\right)}
$$

and

$$
\phi_{2}\left(c_{y}, c_{z}\right)=\left[c_{y} /\left(2^{1 / 2} \pi\right)\right]\left(c_{y}^{2}+c_{z}^{2}-2\right) \mathrm{e}^{-\left(c_{y}^{2}+c_{z}^{2}\right)},
$$

such that if we first multiply Eq. (1) by $\phi_{1}\left(c_{y}, c_{z}\right)$ and integrate over all $c_{y}, c_{z}$, and then repeat the same procedure with $\phi_{2}\left(c_{y}, c_{z}\right)$, we obtain two equations which can be written in a vector equation (for $c_{x}=\xi$ ) as

$$
\xi \frac{\partial}{\partial \tau} \mathbf{G}(\tau, \xi)+\varepsilon \mathbf{G}(\tau, \xi)=\varepsilon \int_{-\infty}^{\infty} \psi\left(\xi^{\prime}\right) \mathbf{K}\left(\xi^{\prime}, \xi\right) \mathbf{G}\left(\tau, \xi^{\prime}\right) \mathrm{d} \xi^{\prime}+\mathbf{A}(\xi) .
$$

Here

$$
\psi(\xi)=\pi^{-1 / 2} \mathrm{e}^{-\xi^{2}}
$$

and

$$
\mathbf{G}(\tau, \xi)=\left[\begin{array}{l}
g_{1}(\tau, \xi) \\
g_{2}(\tau, \xi)
\end{array}\right] .
$$

The components, defined in terms of the parameters $\beta$ and $\varpi$, of the $2 \times 2$ matrix $\mathbf{K}\left(\xi^{\prime}, \xi\right)$ are given by

$$
\begin{gathered}
k_{11}\left(\xi^{\prime}, \xi\right)=1+(2 \beta / 5)\left(\xi^{2}-1 / 2\right)\left(\xi^{2}-1 / 2\right)+2 \varpi \xi^{\prime} \xi \\
k_{12}\left(\xi^{\prime}, \xi\right)=\left(2^{3 / 2} \beta / 5\right)\left(\xi^{2}-1 / 2\right)
\end{gathered}
$$




$$
k_{21}\left(\xi^{\prime}, \xi\right)=\left(2^{3 / 2} \beta / 5\right)\left(\xi^{\prime 2}-1 / 2\right)
$$

and

$$
k_{22}\left(\xi^{\prime}, \xi\right)=4 \beta / 5 .
$$

In addition, for each specific problem, the components of the $2 \times 1$ vector $\mathbf{A}(\xi)$ will be defined by

$$
a_{1}(\xi)=\int_{-\infty}^{\infty} \int_{-\infty}^{\infty} \phi_{1}\left(c_{y}, c_{z}\right) S\left(c_{x}, c_{y}, c_{z}\right) \mathrm{d} c_{y} \mathrm{~d} c_{z}
$$

and

$$
a_{2}(\xi)=\int_{-\infty}^{\infty} \int_{-\infty}^{\infty} \phi_{2}\left(c_{y}, c_{z}\right) S\left(c_{x}, c_{y}, c_{z}\right) \mathrm{d} c_{y} \mathrm{~d} c_{z} .
$$

The same procedure described above is applied to the boundary conditions, Eqs. (9). The resulting boundary conditions, for the $\mathbf{G}(\tau, \xi)$ problem are expressed, for $\xi>0$, as

$$
\mathbf{G}(-a, \xi)-\left(1-\alpha_{1}\right) \mathbf{G}(-a,-\xi)=\mathbf{0}
$$

and

$$
\mathbf{G}(a,-\xi)-\left(1-\alpha_{2}\right) \mathbf{G}(a, \xi)=\mathbf{0} .
$$

Still, from Eqs. (10) and (11), following the same procedure, we find expressions, in terms of the $\mathbf{G}$ solution, for the velocity and heat flow profile, respectively,

$$
\begin{gathered}
u(\tau)=\int_{-\infty}^{\infty} \psi(\xi)\left[\begin{array}{c}
1 \\
0
\end{array}\right]^{T} \mathbf{G}(\tau, \xi) \mathrm{d} \xi \\
q(\tau)=\int_{-\infty}^{\infty} \psi(\xi)\left[\begin{array}{c}
\xi^{2}-1 / 2 \\
2^{1 / 2}
\end{array}\right]^{T} \mathbf{G}(\tau, \xi) \mathrm{d} \xi .
\end{gathered}
$$

We note that $T$ denotes the transpose operation.

In general, Eqs. (18) to (23) define the basic problem to be solved by the ADO method, in order to describe the behavior of a gas when all the models given by Eqs. (5) to (8) are considered. Next, we indicate some special aspects to be taking into account in solving specific well known problems, which we briefly introduce.

\section{The Poiseuille flow problem}

When the flow in a channel, defined by plates located at $\tau=\mp a$, is caused by a (dimensionless) constant pressure gradient, in the direction parallel to the walls, the inhomogeneous term, in Eq. (1) happens to be [21, 29]

$$
S(\mathbf{c})=-k_{P} c_{y},
$$

with $k_{P}$ constant. In this way, we substitute Eq. (26) into Eqs. (22), to define the inhomogeneous vector term in Eq. (18), for the Poiseuille flow problem, as

$$
\mathbf{A}(\xi)=-k_{P}\left[\begin{array}{c}
1 / 2 \\
0
\end{array}\right] .
$$




\section{The thermal-creep problem}

In this case [21, 29], in Eq. (1),

$$
S(\mathbf{c})=-c_{y}\left(c_{x}^{2}+c_{y}^{2}+c_{z}^{2}-5 / 2\right) k_{T},
$$

and $k_{T}$ is a constant gradient of temperature, in the direction parallel to the walls, which induces the flow. Here, we evaluate Eqs. (22), to obtain

$$
\mathbf{A}(\xi)=-\frac{k_{T}}{2}\left[\begin{array}{c}
\xi^{2}-1 / 2 \\
2^{1 / 2}
\end{array}\right]
$$

\section{The Couette flow problem}

When the flow in the channel is driven by plates located at $\tau=\mp a$, moving with velocities, respectively, $u_{w i}, i=1,2$, the linearization process [21] leads to $S(\mathbf{c})=0$, in Eq. (1). In fact, the effect of the velocities of the plates is expressed in the inhomogeneous boundary conditions, now written as

$$
\begin{aligned}
h\left(-a, c_{x}, c_{y}, c_{z}\right)- & \left(1-\alpha_{1}\right) h\left(-a,-c_{x}, c_{y}, c_{z}\right)=2 \alpha_{1} u_{w 1} c_{y}+ \\
& \frac{2 \alpha_{1}}{\pi} \int_{-\infty}^{\infty} \int_{-\infty}^{\infty} \int_{0}^{\infty} \mathrm{e}^{-c^{\prime 2}} h\left(-a,-c_{x}^{\prime}, c_{y}^{\prime}, c_{z}^{\prime}\right) c_{x}^{\prime} \mathrm{d} c_{x}^{\prime} \mathrm{d} c_{y}^{\prime} \mathrm{d} c_{z}^{\prime}
\end{aligned}
$$

and

$$
\begin{aligned}
& h\left(a,-c_{x}, c_{y}, c_{z}\right)-\left(1-\alpha_{2}\right) h\left(a, c_{x}, c_{y}, c_{z}\right)=2 \alpha_{2} u_{w 2} c_{y}+ \\
& \frac{2 \alpha_{2}}{\pi} \int_{-\infty}^{\infty} \int_{-\infty}^{\infty} \int_{0}^{\infty} \mathrm{e}^{-c^{\prime 2}} h\left(a, c_{x}^{\prime}, c_{y}^{\prime}, c_{z}^{\prime}\right) c_{x}^{\prime} \mathrm{d} c_{x}^{\prime} \mathrm{d} c_{y}^{\prime} \mathrm{d} c_{z}^{\prime},
\end{aligned}
$$

where, as previously, $\alpha_{i} \in(0,1], i=1,2$, are the accommodation coefficients.

In this way, in Eq. (18), for the Couette flow problem, we have $\mathbf{A}(\xi)=\mathbf{0}$, while Eqs. (23) are rewritten, for $\xi>0$, as

$$
\mathbf{G}(-a, \xi)-\left(1-\alpha_{1}\right) \mathbf{G}(-a,-\xi)=\alpha_{1} u_{w 1} \boldsymbol{\Omega}
$$

and

$$
\mathbf{G}(a,-\xi)-\left(1-\alpha_{2}\right) \mathbf{G}(a, \xi)=\alpha_{2} u_{w 2} \boldsymbol{\Omega},
$$

with

$$
\boldsymbol{\Omega}=\left[\begin{array}{l}
1 \\
0
\end{array}\right] .
$$

In addition, in this problem, we also want to evaluate a component of the pressure tensor

$$
P_{x y}=\pi^{-1} \int_{-\infty}^{\infty} \int_{-\infty}^{\infty} \int_{-\infty}^{\infty} \mathrm{e}^{-c^{2}} h(\tau, \mathbf{c}) c_{x} c_{y} \mathrm{~d} c_{x} \mathrm{~d} c_{y} \mathrm{~d} c_{z}
$$

which is expressed in the vector form as

$$
P_{x y}=\pi^{1 / 2} \int_{-\infty}^{\infty} \psi(\xi)\left[\begin{array}{l}
1 \\
0
\end{array}\right]^{T} \mathbf{G}(\tau, \xi) \xi \mathrm{d} \xi .
$$




\section{The thermal-slip problem}

In order to define the usually called slip conditions for the Navier-Stokes equations, when the gas has a moderate state of rarefaction, we solve a half-space problem, $\tau \in[0, \infty)$, with

$$
S(\mathbf{c})=-c_{y}\left(c_{x}^{2}+c_{y}^{2}+c_{z}^{2}-5 / 2\right) k_{T}
$$

where, again, $k_{T}$ is a constant temperature gradient in the direction of the flow. Following the same steps described previously, we find in Eq. (18), for this problem,

$$
\mathbf{A}(\xi)=-\frac{k_{T}}{2}\left[\begin{array}{c}
\xi^{2}-1 / 2 \\
2^{1 / 2}
\end{array}\right]
$$

Now, when solving half-space problems, $\tau \in[0, \infty)$, it is usual to consider the plate located at $\tau=0$, such that, for $c_{x}>0$, a boundary condition analogous to Eq. (9a), is written as

$$
\begin{aligned}
h\left(0, c_{x}, c_{y}, c_{z}\right)-(1 & -\alpha) h\left(0,-c_{x}, c_{y}, c_{z}\right) \\
& =\frac{2 \alpha}{\pi} \int_{-\infty}^{\infty} \int_{-\infty}^{\infty} \int_{0}^{\infty} \mathrm{e}^{-c^{\prime 2}} h\left(0,-c_{x}^{\prime}, c_{y}^{\prime}, c_{z}^{\prime}\right) c_{x}^{\prime} \mathrm{d} c_{x}^{\prime} \mathrm{d} c_{y}^{\prime} \mathrm{d} c_{z}^{\prime} .
\end{aligned}
$$

In this case, in addition to a boundary condition, written in a vector form as

$$
\mathbf{G}(0, \xi)-(1-\alpha) \mathbf{G}(0,-\xi)=\mathbf{0}, \quad \xi>0,
$$

the definition of the problem is completed by some given condition at infinity. Here we consider the desired behavior of the velocity

$$
\lim _{\tau \rightarrow \infty} u(\tau)=A_{s}
$$

where $A_{s}$ is a constant, the thermal-slip coefficient, and the velocity profile is defined in Eq. (24).

\section{The viscous-slip problem}

For this half-space problem, $\tau \in[0, \infty)$, in Eq. (1), we find [21]

$$
S(\mathbf{c})=-2 c_{x} c_{y} k_{0}
$$

where $k_{0}$ is a constant velocity gradient in the normal direction of the flow. In this way, in Eq. (18)

$$
\mathbf{A}(\xi)=-k_{0}\left[\begin{array}{l}
\xi \\
0
\end{array}\right]
$$

In addition to the condition

$$
\mathbf{G}(0, \xi)-(1-\alpha) \mathbf{G}(0,-\xi)=\mathbf{0}, \quad \xi>0
$$

we consider the velocity profile, defined as

$$
u_{k}(\tau)=k_{0} \tau+\int_{-\infty}^{\infty} \psi(\xi)\left[\begin{array}{l}
1 \\
0
\end{array}\right]^{T} \mathbf{G}(\tau, \xi) \mathrm{d} \xi
$$


diverges at infinity, but in a way such that

$$
\lim _{\tau \rightarrow \infty} \frac{\mathrm{d}}{\mathrm{d} \tau} u_{k}(\tau)=k_{0}
$$

\section{General solution}

In the previous section we showed that the basic problem, the $\mathbf{G}$ problem, to be solved in most of the cases related to the problems of interest, defined by Eqs. (18) to (23), has a source term. In this way, the general solution of the problem has to be proposed in a way that

$$
\mathbf{G}(\tau, \xi)=\mathbf{G}^{h}(\tau, \xi)+\mathbf{G}^{p}(\tau, \xi)
$$

where the particular solution $\mathbf{G}^{p}(\tau, \xi)$ has to be found for each specific problem.

Here, we follow previous works $[32,27]$ and we propose, for Eq. (18), particular solutions of the form

$$
\mathbf{G}^{p}(\tau, \xi)=\mathbf{B} \tau^{2}+\mathbf{C} \tau \xi+\mathbf{D} \xi^{2}+\mathbf{E} \xi+\mathbf{F}
$$

where $\mathbf{B}, \mathbf{C}, \mathbf{D}, \mathbf{E}$ and $\mathbf{F}$ are $2 \times 1$ constant vectors. Then, if we substitute Eq. (46) into Eq. (18), we find, after noting Eqs. (27),(29),(36) and (41), respectively,

$$
\mathbf{G}^{p}(\tau, \xi)=k_{P}\left[\begin{array}{c}
\varepsilon(1-\varpi) \tau^{2} / 2-\tau \xi+\left[(5-4 \beta) \xi^{2}\right] /[5 \varepsilon(1-\beta)] \\
2^{1 / 2} \beta /[5 \varepsilon(1-\beta)]
\end{array}\right],
$$

for the Poiseuille flow problem;

$$
\mathbf{G}^{p}(\tau, \xi)=\frac{k_{T}}{2 \varepsilon(\beta-1)}\left[\begin{array}{c}
\xi^{2} \\
2^{1 / 2}
\end{array}\right],
$$

for the thermal-creep and thermal-slip problems; and

$$
\mathbf{G}^{p}(\tau, \xi)=\frac{k_{0} \xi}{\varepsilon(\varpi-1)}\left[\begin{array}{l}
1 \\
0
\end{array}\right]
$$

for the viscous-slip problem.

Looking back to Eq. (45), we note that, when we substitute that equation into Eq. (18) we find that the homogeneous solution, $\mathbf{G}^{h}(\tau, \xi)$ must satisfy the following problem:

$$
\xi \frac{\partial}{\partial \tau} \mathbf{G}^{h}(\tau, \xi)+\varepsilon \mathbf{G}^{h}(\tau, \xi)=\varepsilon \int_{-\infty}^{\infty} \psi\left(\xi^{\prime}\right) \mathbf{K}\left(\xi^{\prime}, \xi\right) \mathbf{G}^{h}\left(\tau, \xi^{\prime}\right) \mathrm{d} \xi^{\prime} .
$$

Since Eq. (45) is used also in the boundary conditions, we obtain, for the homogeneous problem the boundary equations written, for $\xi>0$, as

$$
\mathbf{G}^{h}(-a, \xi)-\left(1-\alpha_{1}\right) \mathbf{G}^{h}(-a,-\xi)=\mathbf{R}_{+}(\xi)
$$

and

$$
\mathbf{G}^{h}(a,-\xi)-\left(1-\alpha_{2}\right) \mathbf{G}^{h}(a, \xi)=\mathbf{R}_{-}(\xi),
$$


for the Poiseuille and thermal-creep (plane channel) problems. Here we note that

$$
\mathbf{R}_{+}(\xi)=\left(1-\alpha_{1}\right) \mathbf{G}^{p}(-a,-\xi)-\mathbf{G}^{p}(-a, \xi)
$$

and

$$
\mathbf{R}_{-}(\xi)=\left(1-\alpha_{2}\right) \mathbf{G}^{p}(a, \xi)-\mathbf{G}^{p}(a,-\xi) .
$$

On the other hand, for the Couette flow problem, which is already an homogeneous problem $(\mathbf{A}(\xi)=0)$, and we do not have to compute a particular solution. In other words, in Eq. (45), $\mathbf{G}^{p}(\tau, \xi)=\mathbf{0}$. In this problem, noting Eqs. (31), the boundary conditions, Eqs. (51), are such that

$$
\mathbf{R}_{+}(\xi)=\alpha_{1} u_{w 1}\left[\begin{array}{l}
1 \\
0
\end{array}\right]
$$

and

$$
\mathbf{R}_{-}(\xi)=\alpha_{2} u_{w 2}\left[\begin{array}{l}
1 \\
0
\end{array}\right] .
$$

For the half-space problems, when we substitute Eq. (45) into Eqs. (38) and (42), we obtain, for $\xi>0$,

$$
\mathbf{G}^{h}(0, \xi)-(1-\alpha) \mathbf{G}^{h}(0,-\xi)=\mathbf{R}(\xi)
$$

with

$$
\mathbf{R}(\xi)=(1-\alpha) \mathbf{G}^{p}(0,-\xi)-\mathbf{G}^{p}(0, \xi) .
$$

In the next section we proceed to develop a discrete ordinates solution for the problem defined by Eq. (50).

\section{A discrete ordinates solution}

In order to develop a solution for the homogeneous problem, defined by Eq. (50), we first note that $\psi(\xi)$, Eq. (19), is an even function, and we rewrite Eq. (50) as

$$
\begin{aligned}
\xi \frac{\partial}{\partial \tau} \mathbf{G}^{h}(\tau, \xi) & +\varepsilon \mathbf{G}^{h}(\tau, \xi) \\
& =\varepsilon \int_{0}^{\infty} \psi\left(\xi^{\prime}\right)\left[\mathbf{K}\left(\xi^{\prime}, \xi\right) \mathbf{G}^{h}\left(\tau, \xi^{\prime}\right)+\mathbf{K}\left(-\xi^{\prime}, \xi\right) \mathbf{G}^{h}\left(\tau,-\xi^{\prime}\right)\right] \mathrm{d} \xi^{\prime} .
\end{aligned}
$$

We then introduce a half-range quadrature scheme to deal with the integral term of Eq. (55), which we express now as

$$
\begin{aligned}
\xi \frac{\partial}{\partial \tau} \mathbf{G}^{h}(\tau, \xi) & +\varepsilon \mathbf{G}^{h}(\tau, \xi) \\
& =\varepsilon \sum_{k=1}^{N} \omega_{k} \psi\left(\xi_{k}\right)\left[\mathbf{K}\left(\xi_{k}, \xi\right) \mathbf{G}^{h}\left(\tau, \xi_{k}\right)+\mathbf{K}\left(-\xi_{k}, \xi\right) \mathbf{G}^{h}\left(\tau,-\xi_{k}\right)\right]
\end{aligned}
$$


where $\left\{\xi_{k}, \omega_{k}\right\}$ are the $N$ arbitrary nodes and weights defined for the interval $[0, \infty)$. In this way, we assume $\xi= \pm \xi_{i}$, for $i=1, \ldots, N$, in Eq. (56) to obtain the discrete ordinates version of Eq. (55),

$$
\begin{aligned}
& \pm \xi_{i} \frac{\mathrm{d}}{\mathrm{d} \tau} \mathbf{G}^{h}\left(\tau, \pm \xi_{i}\right)+\varepsilon \mathbf{G}^{h}\left(\tau, \pm \xi_{i}\right) \\
& \quad=\varepsilon \sum_{k=1}^{N} \omega_{k} \psi\left(\xi_{k}\right)\left[\mathbf{K}\left(\xi_{k}, \pm \xi_{i}\right) \mathbf{G}^{h}\left(\tau, \xi_{k}\right)+\mathbf{K}\left(-\xi_{k}, \pm \xi_{i}\right) \mathbf{G}^{h}\left(\tau,-\xi_{k}\right)\right]
\end{aligned}
$$

We now seek for exponential solutions of the system given by Eq. (57), in the form

$$
\mathbf{G}^{h}(\tau, \xi)=\mathbf{\Phi}(\nu, \xi) \mathrm{e}^{-\varepsilon \tau / \nu},
$$

where

$$
\boldsymbol{\Phi}(\nu, \xi)=\left[\begin{array}{c}
\Phi_{1}(\nu, \xi) \\
\Phi_{2}(\nu, \xi)
\end{array}\right] .
$$

We then substitute Eq. (58) into Eq. (57) to obtain, for $i=1, \ldots, N$,

$$
\left(1 \mp \xi_{i} / \nu\right) \boldsymbol{\Phi}\left(\nu, \pm \xi_{i}\right)=\sum_{k=1}^{N} \omega_{k} \psi\left(\xi_{k}\right)\left[\mathbf{K}\left(\xi_{k}, \pm \xi_{i}\right) \mathbf{\Phi}\left(\nu, \xi_{k}\right)+\mathbf{K}\left(-\xi_{k}, \pm \xi_{i}\right) \boldsymbol{\Phi}\left(\nu,-\xi_{k}\right)\right],
$$

which we write in a matrix form as

$$
\left(\mathbf{I}-\mathbf{M} \nu^{-1}\right) \boldsymbol{\Phi}_{+}(\nu)=\mathbf{W}(+,+) \boldsymbol{\Phi}_{+}(\nu)+\mathbf{W}(-,+) \boldsymbol{\Phi}_{-}(\nu)
$$

and

$$
\left(\mathbf{I}+\mathbf{M} \nu^{-1}\right) \boldsymbol{\Phi}_{-}(\nu)=\mathbf{W}(+,-) \boldsymbol{\Phi}_{+}(\nu)+\mathbf{W}(-,-) \boldsymbol{\Phi}_{-}(\nu),
$$

with $\mathbf{I}$ the $2 N \times 2 N$ identity matrix, $\mathbf{M}$ the $2 N \times 2 N$ matrix defined as

$$
\mathbf{M}=\operatorname{diag}\left\{\xi_{1}, \xi_{2}, \ldots, \xi_{N}, \xi_{1}, \xi_{2}, \ldots, \xi_{N}\right\},
$$

and $\boldsymbol{\Phi}_{ \pm}(\nu)$ the $2 N \times 1$ vectors

$$
\boldsymbol{\Phi}_{ \pm}(\nu)=\left[\begin{array}{llllll}
\Phi_{1}\left(\nu, \pm \xi_{1}\right) & \cdots & \Phi_{1}\left(\nu, \pm \xi_{N}\right) & \Phi_{2}\left(\nu, \pm \xi_{1}\right) & \cdots & \Phi_{2}\left(\nu, \pm \xi_{N}\right)
\end{array}\right]^{T} .
$$

Here $T$ denotes the transpose operation. Continuing, $\mathbf{W}( \pm, \pm)$ is a $2 N \times 2 N$ matrix

$$
\mathbf{W}( \pm, \pm)=\left[\begin{array}{ll}
\mathbf{W}_{11}( \pm, \pm) & \mathbf{W}_{\mathbf{1 2}}( \pm, \pm) \\
\mathbf{W}_{\mathbf{2 1}}( \pm, \pm) & \mathbf{W}_{\mathbf{2 2}}( \pm, \pm)
\end{array}\right]
$$

which have $N \times N$ sub-matrices as components, defined by

$$
\left[\mathbf{W}_{m n}( \pm, \pm)\right]_{i, j}=\omega_{j} \psi\left(\xi_{j}\right) k_{m n}\left( \pm \xi_{j}, \pm \xi_{i}\right)
$$

for $m, n=1,2$ and $i, j=1, \ldots, N$. Here $\omega_{j}$ are the weights of the quadrature scheme, $\psi\left(\xi_{j}\right)$ is defined in Eq. (19) and $k_{m n}\left( \pm \xi_{j}, \pm \xi_{i}\right)$ are the components given 
in Eqs. (21). In fact, we can see from Eqs. (21) that the $2 \times 2$ matrix $\mathbf{K}\left(\xi^{\prime}, \xi\right)$ is such that

$$
\mathbf{K}\left(\xi^{\prime}, \xi\right)=\mathbf{K}\left(-\xi^{\prime},-\xi\right)
$$

and

$$
\mathbf{K}\left(-\xi^{\prime}, \xi\right)=\mathbf{K}\left(\xi^{\prime},-\xi\right)
$$

So, if we consider the result given by Eqs. (66) and note Eq. (65), we can verify that, in Eqs. (64),

$$
\mathbf{W}(+,+)=\mathbf{W}(-,-)
$$

and

$$
\mathbf{W}(+,-)=\mathbf{W}(-,+) .
$$

In this way, from now on, we use

$$
\mathbf{W}_{+}=\mathbf{W}(+,+)=\mathbf{W}(-,-)
$$

and

$$
\mathbf{W}_{-}=\mathbf{W}(+,-)=\mathbf{W}(-,+) .
$$

We use Eqs. (68) to rewrite Eqs. (61) as

$$
\left(\mathbf{I}-\mathbf{M} \nu^{-1}\right) \boldsymbol{\Phi}_{+}(\nu)=\mathbf{W}_{+} \boldsymbol{\Phi}_{+}(\nu)+\mathbf{W}_{-} \boldsymbol{\Phi}_{-}(\nu)
$$

and

$$
\left(\mathbf{I}+\mathbf{M} \nu^{-1}\right) \boldsymbol{\Phi}_{-}(\nu)=\mathbf{W}_{-} \boldsymbol{\Phi}_{+}(\nu)+\mathbf{W}_{+} \boldsymbol{\Phi}_{-}(\nu) .
$$

Now, we let

$$
\mathbf{U}=\boldsymbol{\Phi}_{+}(\nu)+\boldsymbol{\Phi}_{-}(\nu),
$$

where $\boldsymbol{\Phi}_{+}(\nu)$ and $\boldsymbol{\Phi}_{-}(\nu)$ are $2 N \times 1$ vectors defined in Eq. (63), and we add and subtract Eqs. (69) to derive an eigenvalue problem

$$
\mathbf{A X}=\lambda \mathbf{X}
$$

where $\mathbf{A}$ is a $2 N \times 2 N$ matrix defined as

$$
\mathbf{A}=\left(\mathbf{W}_{+}-\mathbf{W}_{-}-\mathbf{I}\right) \mathbf{M}^{-\mathbf{1}}\left(\mathbf{W}_{+}+\mathbf{W}_{-}-\mathbf{I}\right) \mathbf{M}^{-\mathbf{1}},
$$

$\mathbf{X}$ is a $2 N \times 1$ vector, such that

$$
\mathbf{X}=\mathbf{M U}
$$

and, the eigenvalues are related to the separation constants, in Eq. (58), as

$$
\lambda=\nu^{-2} .
$$

In fact, from Eq. (71) we obtain $2 N$ separation constants $\nu_{j}$ and $2 N$ eigenvectors $\mathbf{X}\left(\nu_{j}\right)$, which we use, along with Eqs. (61) to write the $2 N \times 1$ elementary solutions

$$
\mathbf{\Phi}_{+}\left(\nu_{j}\right)=\frac{1}{2} \mathbf{M}^{-\mathbf{1}}\left[\mathbf{I}-\nu_{j}\left(\mathbf{W}_{+}+\mathbf{W}_{-}-\mathbf{I}\right) \mathbf{M}^{-\mathbf{1}}\right] \mathbf{X}\left(\nu_{j}\right)
$$


and

$$
\mathbf{\Phi}_{-}\left(\nu_{j}\right)=\frac{1}{2} \mathbf{M}^{-\mathbf{1}}\left[\mathbf{I}+\nu_{j}\left(\mathbf{W}_{+}+\mathbf{W}_{-}-\mathbf{I}\right) \mathbf{M}^{-\mathbf{1}}\right] \mathbf{X}\left(\nu_{j}\right) .
$$

Looking back to Eq. (58), we are able now to write the general solution of the problem given by Eq. (56) as

$$
\mathbf{G}^{h}\left(\tau, \pm \xi_{i}\right)=\sum_{j=1}^{2 N}\left[A_{j} \boldsymbol{\Phi}\left(\nu_{j}, \pm \xi_{i}\right) \mathrm{e}^{-\varepsilon(a+\tau) / \nu_{j}}+B_{j} \boldsymbol{\Phi}\left(\nu_{j}, \mp \xi_{i}\right) \mathrm{e}^{-\varepsilon(a-\tau) / \nu_{j}}\right]
$$

At this point, as usual for conservative problems, we have to consider that some, in this case just one, eigenvalues go to zero when $N$ goes to infinity, and we have to introduce some exact solutions. Here, we take this aspect into account and we rewrite Eq. (76), as

$$
\begin{aligned}
\mathbf{G}^{h}\left(\tau, \pm \xi_{i}\right)= & A_{1}^{*} \mathbf{G}_{1}+B_{1}^{*} \mathbf{G}_{2}\left(\tau, \pm \xi_{i}\right) \\
& +\sum_{j=1}^{2 N-1}\left[A_{j} \boldsymbol{\Phi}\left(\nu_{j}, \pm \xi_{i}\right) \mathrm{e}^{-\varepsilon(a+\tau) / \nu_{j}}+B_{j} \boldsymbol{\Phi}\left(\nu_{j}, \mp \xi_{i}\right) \mathrm{e}^{-\varepsilon(a-\tau) / \nu_{j}}\right]
\end{aligned}
$$

for the plane channel problems, and assuming $a=0$

$$
\begin{aligned}
\mathbf{G}^{h}\left(\tau, \pm \xi_{i}\right)=A_{1}^{*} \mathbf{G}_{1} & +B_{1}^{*} \mathbf{G}_{2}\left(\tau, \pm \xi_{i}\right) \\
& +\sum_{j=1}^{2 N-1}\left[A_{j} \mathbf{\Phi}\left(\nu_{j}, \pm \xi_{i}\right) \mathrm{e}^{-\varepsilon \tau / \nu_{j}}+B_{j} \boldsymbol{\Phi}\left(\nu_{j}, \mp \xi_{i}\right) \mathrm{e}^{\varepsilon \tau / \nu_{j}}\right],
\end{aligned}
$$

for the half-space problems. In these expressions, the chosen exact solutions of the problem given by Eq. (55) are

$$
\mathbf{G}_{1}=\left[\begin{array}{l}
1 \\
0
\end{array}\right]
$$

and

$$
\mathbf{G}_{2}(\tau, \xi)=\left[\begin{array}{c}
\varepsilon \tau-\xi /(1-\varpi) \\
0
\end{array}\right]
$$

We proceed to evaluate the $4 N$ coefficients $A_{1}^{*}, B_{1}^{*}, A_{j}, B_{j}, j=1, \ldots, 2 N-1$ to establish the complete $\mathrm{ADO}$ solution and to evaluate the quantities of interest.

\section{Physical quantities of interest}

At this point we need to apply the proposed form of the general solution, Eqs. (77) and (78), respectively, for plane channel and half-space problems, into the boundary equations, in order to determine the arbitrary constants present in the solution and, next, to evaluate the velocity and heat flow profiles, as well as the other quantities of interest. 
For channel problems, we look back to Eqs. (51) where we then substitute Eq. (77), such that, for $i=1, \ldots, N$ we obtain a $4 N \times 4 N$ linear system,

$$
\begin{aligned}
\sum_{j=1}^{2 N-1}\left\{A _ { j } \left[\boldsymbol { \Phi } \left(\nu_{j},\right.\right.\right. & \left.\left.\xi_{i}\right)-\left(1-\alpha_{1}\right) \boldsymbol{\Phi}\left(\nu_{j},-\xi_{i}\right)\right] \\
+ & \left.B_{j}\left[\boldsymbol{\Phi}\left(\nu_{j},-\xi_{i}\right)-\left(1-\alpha_{1}\right) \boldsymbol{\Phi}\left(\nu_{j}, \xi_{i}\right)\right] \mathrm{e}^{-2 a \varepsilon / \nu_{j}}\right\} \\
& +A_{1}^{*} \alpha_{1} \mathbf{G}_{1}-B_{1}^{*}\left[a \varepsilon \alpha_{1} \mathbf{G}_{1}+\left(\alpha_{1}-2\right) \mathbf{G}_{2}\left(0, \xi_{i}\right)\right]=\mathbf{R}_{+}\left(\xi_{i}\right)
\end{aligned}
$$

and

$$
\begin{aligned}
\sum_{j=1}^{2 N-1}\left\{A _ { j } \left[\boldsymbol{\Phi}\left(\nu_{j},-\xi_{i}\right)\right.\right. & \left.-\left(1-\alpha_{2}\right) \boldsymbol{\Phi}\left(\nu_{j}, \xi_{i}\right)\right] \mathrm{e}^{-2 a \varepsilon / \nu_{j}} \\
& \left.+B_{j}\left[\mathbf{\Phi}\left(\nu_{j}, \xi_{i}\right)-\left(1-\alpha_{2}\right) \mathbf{\Phi}\left(\nu_{j},-\xi_{i}\right)\right]\right\} \\
+ & A_{1}^{*} \alpha_{2} \mathbf{G}_{1}+B_{1}^{*}\left[a \varepsilon \alpha_{2} \mathbf{G}_{1}+\left(\alpha_{2}-2\right) \mathbf{G}_{2}\left(0, \xi_{i}\right)\right]=\mathbf{R}_{-}\left(\xi_{i}\right) .
\end{aligned}
$$

The right-hand side of Eqs. (81) is expressed by different forms depending on the problem to be solved, according Eqs. (52) and (53). In this sense, for the Poiseuille flow problem

$$
\begin{aligned}
& \mathbf{R}_{+}\left(\xi_{i}\right)=\mathbf{R}_{P+}\left(\xi_{i}\right) \\
& \quad=k_{P}\left[\begin{array}{c}
\left(\alpha_{1}-2\right) a \xi_{i}-\alpha_{1} \varepsilon(1-\varpi) a^{2} / 2-\left[\alpha_{1}(5-4 \beta) \xi_{i}^{2}\right] /[5 \varepsilon(1-\beta)] \\
-\alpha_{1} 2^{1 / 2} \beta /[5 \varepsilon(1-\beta)]
\end{array}\right]
\end{aligned}
$$

and

$$
\begin{aligned}
& \mathbf{R}_{-}\left(\xi_{i}\right)=\mathbf{R}_{P-}\left(\xi_{i}\right) \\
& \quad=k_{P}\left[\begin{array}{c}
\left(\alpha_{2}-2\right) a \xi_{i}-\alpha_{2} \varepsilon(1-\varpi) a^{2} / 2-\left[\alpha_{2}(5-4 \beta) \xi_{i}^{2}\right] /[5 \varepsilon(1-\beta)] \\
-\alpha_{2} 2^{1 / 2} \beta /[5 \varepsilon(1-\beta)]
\end{array}\right] .
\end{aligned}
$$

Differently, for the thermal-creep problem,

$$
\mathbf{R}_{+}\left(\xi_{i}\right)=\mathbf{R}_{T+}\left(\xi_{i}\right)=\frac{k_{T} \alpha_{1}}{2 \varepsilon(1-\beta)}\left[\begin{array}{c}
\xi_{i}^{2} \\
2^{1 / 2}
\end{array}\right]
$$

and

$$
\mathbf{R}_{-}\left(\xi_{i}\right)=\mathbf{R}_{T-}\left(\xi_{i}\right)=\frac{k_{T} \alpha_{2}}{2 \varepsilon(1-\beta)}\left[\begin{array}{c}
\xi_{i}^{2} \\
2^{1 / 2}
\end{array}\right]
$$

Finally, for solving the Couette flow problem, we have to evaluate the system given by Eqs. (81) with the right-hand side given by

$$
\mathbf{R}_{+}\left(\xi_{i}\right)=\mathbf{R}_{C+}\left(\xi_{i}\right)=\alpha_{1} u_{w 1}\left[\begin{array}{l}
1 \\
0
\end{array}\right]
$$

and

$$
\mathbf{R}_{-}\left(\xi_{i}\right)=\mathbf{R}_{C-}\left(\xi_{i}\right)=\alpha_{2} u_{w 2}\left[\begin{array}{l}
1 \\
0
\end{array}\right]
$$


for $i=1, \ldots, N$.

In this way, looking back to Eqs. (24), (25) and (34), we see that once we have solved the $\mathbf{G}$ problem and we have completely established the solution, given by Eq. (45), we can use the definitions

$$
\mathbf{V}\left(\nu_{j}\right)=\sum_{k=1}^{N} \omega_{k} \psi\left(\xi_{k}\right)\left[\begin{array}{l}
1 \\
0
\end{array}\right]^{T}\left[\mathbf{\Phi}\left(\nu_{j}, \xi_{k}\right)+\mathbf{\Phi}\left(\nu_{j},-\xi_{k}\right)\right]
$$

and

$$
\mathbf{Y}\left(\nu_{j}\right)=\sum_{k=1}^{N} \omega_{k} \psi\left(\xi_{k}\right)\left[\begin{array}{c}
\xi_{k}^{2}-1 / 2 \\
2^{1 / 2}
\end{array}\right]^{T}\left[\boldsymbol{\Phi}\left(\nu_{j}, \xi_{k}\right)+\mathbf{\Phi}\left(\nu_{j},-\xi_{k}\right)\right],
$$

to write the following quantities:

\section{Poiseuille flow problem}

- velocity profile

$$
\begin{aligned}
u_{P}(\tau)=k_{P}\left[\frac{\varepsilon(1-\varpi) \tau^{2}}{2}+\right. & \left.\frac{5-4 \beta}{10 \varepsilon(1-\beta)}\right]+A_{1}^{*}+B_{1}^{*} \varepsilon \tau \\
& +\sum_{j=1}^{2 N-1}\left[A_{j} \mathrm{e}^{-\varepsilon(a+\tau) / \nu_{j}}+B_{j} \mathrm{e}^{-\varepsilon(a-\tau) / \nu_{j}}\right] \mathbf{V}\left(\nu_{j}\right)
\end{aligned}
$$

- heat flow profile

$$
q_{P}(\tau)=\frac{k_{P}}{2 \varepsilon(1-\beta)}+\sum_{j=1}^{2 N-1}\left[A_{j} \mathrm{e}^{-\varepsilon(a+\tau) / \nu_{j}}+B_{j} \mathrm{e}^{-\varepsilon(a-\tau) / \nu_{j}}\right] \mathbf{Y}\left(\nu_{j}\right)
$$

- mass flow rate

$$
\begin{aligned}
U_{P}=k_{P}\left[\frac{\varepsilon(1-\varpi) a}{6}+\frac{5-4 \beta}{10 a \varepsilon(1-\beta)}\right] & +\frac{A_{1}^{*}}{a} \\
& +\frac{1}{2 \varepsilon a^{2}} \sum_{j=1}^{2 N-1} \nu_{j}\left(A_{j}+B_{j}\right)\left(1-\mathrm{e}^{-2 a \varepsilon / \nu_{j}}\right) \mathbf{V}\left(\nu_{j}\right)
\end{aligned}
$$

- heat flow rate

$$
Q_{P}=\frac{k_{P}}{2 a \varepsilon(1-\beta)}+\frac{1}{2 \varepsilon a^{2}} \sum_{j=1}^{2 N-1} \nu_{j}\left(A_{j}+B_{j}\right)\left(1-\mathrm{e}^{-2 a \varepsilon / \nu_{j}}\right) \mathbf{Y}\left(\nu_{j}\right) .
$$

\section{Thermal-creep problem}

- velocity profile

$$
u_{T}(\tau)=\frac{k_{T}}{4 \varepsilon(\beta-1)}+A_{1}^{*}+B_{1}^{*} \varepsilon \tau+\sum_{j=1}^{2 N-1}\left[A_{j} \mathrm{e}^{-\varepsilon(a+\tau) / \nu_{j}}+B_{j} \mathrm{e}^{-\varepsilon(a-\tau) / \nu_{j}}\right] \mathbf{V}\left(\nu_{j}\right)
$$


- heat flow profile

$$
q_{T}(\tau)=\frac{5 k_{T}}{4 \varepsilon(\beta-1)}+\sum_{j=1}^{2 N-1}\left[A_{j} \mathrm{e}^{-\varepsilon(a+\tau) / \nu_{j}}+B_{j} \mathrm{e}^{-\varepsilon(a-\tau) / \nu_{j}}\right] \mathbf{Y}\left(\nu_{j}\right)
$$

- mass flow rate

$$
U_{T}=\frac{k_{T}}{4 a \varepsilon(\beta-1)}+\frac{A_{1}^{*}}{a}+\frac{1}{2 \varepsilon a^{2}} \sum_{j=1}^{2 N-1} \nu_{j}\left(A_{j}+B_{j}\right)\left(1-\mathrm{e}^{-2 a \varepsilon / \nu_{j}}\right) \mathbf{V}\left(\nu_{j}\right)
$$

- heat flow rate

$$
Q_{T}=\frac{5 k_{T}}{4 a \varepsilon(\beta-1)}+\frac{1}{2 \varepsilon a^{2}} \sum_{j=1}^{2 N-1} \nu_{j}\left(A_{j}+B_{j}\right)\left(1-\mathrm{e}^{-2 a \varepsilon / \nu_{j}}\right) \mathbf{Y}\left(\nu_{j}\right) .
$$

\section{Couette flow problem}

- velocity profile

$$
u_{C}(\tau)=A_{1}^{*}+B_{1}^{*} \varepsilon \tau+\sum_{j=1}^{2 N-1}\left[A_{j} \mathrm{e}^{-\varepsilon(a+\tau) / \nu_{j}}+B_{j} \mathrm{e}^{-\varepsilon(a-\tau) / \nu_{j}}\right] \mathbf{V}\left(\nu_{j}\right)
$$

- heat flow profile

$$
q_{C}(\tau)=\sum_{j=1}^{2 N-1}\left[A_{j} \mathrm{e}^{-\varepsilon(a+\tau) / \nu_{j}}+B_{j} \mathrm{e}^{-\varepsilon(a-\tau) / \nu_{j}}\right] \mathbf{Y}\left(\nu_{j}\right)
$$

- mass flow rate

$$
\begin{aligned}
U_{C}=\frac{1}{2 a^{2}} \int_{0}^{a} u_{C}(\tau) \mathrm{d} \tau & =\frac{A_{1}^{*}}{2 a}+\frac{B_{1}^{*} \varepsilon}{4} \\
& +\frac{1}{2 \varepsilon a^{2}} \sum_{j=1}^{2 N-1} \nu_{j}\left(A_{j} \mathrm{e}^{-a \varepsilon / \nu_{j}}+B_{j}\right)\left(1-\mathrm{e}^{-a \varepsilon / \nu_{j}}\right) \mathbf{V}\left(\nu_{j}\right)
\end{aligned}
$$

- heat flow rate

$$
Q_{C}=\frac{1}{2 a^{2}} \int_{0}^{a} q_{C}(\tau) \mathrm{d} \tau=\frac{1}{2 \varepsilon a^{2}} \sum_{j=1}^{2 N-1} \nu_{j}\left(A_{j} \mathrm{e}^{-a \varepsilon / \nu_{j}}+B_{j}\right)\left(1-\mathrm{e}^{-a \varepsilon / \nu_{j}}\right) \mathbf{Y}\left(\nu_{j}\right)
$$

- a component of the pressure tensor

$$
P_{x y}=\frac{B_{1}^{*} \pi^{1 / 2}}{2(\varpi-1)} .
$$

Analogously, for half-space problems, we begin with the general solution given by Eq. (78). However, we now choose $B_{1}^{*}=0$ and $B_{j}=0$ for $j=1, \ldots, 2 N-1$, to be 
consistent with the required behavior at infinity. The solution for the homogeneous problem is then expressed as

$$
\mathbf{G}^{h}\left(\tau, \pm \xi_{i}\right)=A_{1}^{*} \mathbf{G}_{1}+\sum_{j=1}^{2 N-1} A_{j} \mathbf{\Phi}\left(\nu_{j}, \pm \xi_{i}\right) \mathrm{e}^{-\varepsilon \tau / \nu_{j}} .
$$

We substitute Eq. (100) into the discrete ordinates version of the boundary condition, given by Eqs. (54), to obtain, for $i=1, \ldots, N$, the $2 N \times 2 N$ linear system

$$
\alpha A_{1}^{*} \mathbf{G}_{1}+\sum_{j=1}^{2 N-1} A_{j}\left[\mathbf{\Phi}\left(\nu_{j}, \xi_{i}\right)-(1-\alpha) \mathbf{\Phi}\left(\nu_{j},-\xi_{i}\right)\right]=\mathbf{R}\left(\xi_{i}\right),
$$

where, for the thermal-slip problem

$$
\mathbf{R}\left(\xi_{i}\right)=\mathbf{R}_{s}\left(\xi_{i}\right)=\frac{k_{T} \alpha}{2 \varepsilon(1-\beta)}\left[\begin{array}{c}
\xi_{i}^{2} \\
2^{1 / 2}
\end{array}\right]
$$

and for the viscous-slip problem

$$
\mathbf{R}\left(\xi_{i}\right)=\mathbf{R}_{k}\left(\xi_{i}\right)=\frac{k_{0}(\alpha-2) \xi_{i}}{\varepsilon(\varpi-1)}\left[\begin{array}{l}
1 \\
0
\end{array}\right] .
$$

Once we solve the system, Eq. (101), we are in position of evaluating the following expressions:

\section{Thermal-slip problem}

- velocity profile

$$
u_{s}(\tau)=\frac{k_{T}}{4 \varepsilon(\beta-1)}+A_{1}^{*}+\sum_{j=1}^{2 N-1} A_{j} \mathrm{e}^{-\varepsilon \tau / \nu_{j}} \mathbf{V}\left(\nu_{j}\right)
$$

- heat flow profile

$$
q_{s}(\tau)=\frac{5 k_{T}}{4 \varepsilon(\beta-1)}+\sum_{j=1}^{2 N-1} A_{j} \mathrm{e}^{-\varepsilon \tau / \nu_{j}} \mathbf{Y}\left(\nu_{j}\right)
$$

- thermal-slip coefficient

$$
A_{s}=\frac{k_{T}}{4 \varepsilon(\beta-1)}+A_{1}^{*} .
$$

\section{Viscous-slip problem}

- velocity profile

$$
u_{k}(\tau)=k_{0} \tau+A_{1}^{*}+\sum_{j=1}^{2 N-1} A_{j} \mathrm{e}^{-\varepsilon \tau / \nu_{j}} \mathbf{V}\left(\nu_{j}\right)
$$


- heat flow profile

$$
q_{k}(\tau)=\sum_{j=1}^{2 N-1} A_{j} \mathrm{e}^{-\varepsilon \tau / \nu_{j}} \mathbf{Y}\left(\nu_{j}\right)
$$

- viscous-slip coefficient $A_{k}$ is such that

$$
u_{a s y}(0)=\left.A_{k} \frac{\mathrm{d}}{\mathrm{d} \tau} u_{a s y}(\tau)\right|_{\tau=0},
$$

where

$$
u_{a s y}(\tau)=k_{0} \tau+A_{1}^{*}
$$

In this way,

$$
A_{k}=\frac{A_{1}^{*}}{k_{0}}
$$

\section{Computational aspects and numerical results}

The basic steps to implement the ADO solution developed here are simple:

- to solve the eigenvalue problem given by Eq. (71);

- to evaluate the elementary solutions, Eqs. (75);

- to solve the linear system defined in Eqs. (81), for plane channel problems, and in Eq. (101), for half-space problems;

- to evaluate the quantities - Eqs. (87) to (99) and (104) to (111) - defined in the previous section.

We have done that using FORTRAN programs, where linear algebra software packages, usually LINPACK [36] and EISPACK [37], are used to deal with the eigenvalue problems and the linear system. In this specific work, two independent programs were developed in order to check the results presented.

However, we emphasize that, according with the derivation initiated in Eq. (55), one of the (good) features of the ADO method is the use of an arbitrary quadrature scheme. So, the very first step, needed to define the eigenvalue problem mentioned in the above list, is to define a quadrature scheme. In most of the works related to applications of the ADO method, in rarefied gas dynamics problems, a half-range quadrature scheme is proposed. In fact, we first use the transformation

$$
u(\xi)=\mathrm{e}^{-\xi}
$$

to map the interval $[0, \infty)$ into $[0,1]$ and we then make use of another change of variable,

$$
v(u)=2 u-1,
$$

to be able to work, for $v \in[-1,1]$, with the known Gauss-Legendre quadrature scheme. In this way, we approximate the integral term originally defined in the interval $[0, \infty)$. 
The numerical results listed in the tables were obtained with $N=50$ quadrature points, and, checked by two different programs. In general, it takes less than one second to obtain numerical results for a profile, in a Pentium IV - 2,66 GHz. We have tested many different values for $N$ to have more confidence in our results and we note that the digits listed here are kept (plus or minus one in the last digit) when the value of $N$ increases.

Since we have available an analytical procedure, which generates a fast solution and which easily allow us to work with a wide range of parameters, it seems a good point to have a wide series of results, which may give us possible ideas about physical behavior, and, in particular to establish a general view of the different kinetic models results.

Then, we present in Tables 1 to 26 results for all the problems described in Sec. 3. First of all, to have more confidence in our programs, by an appropriate choice of the parameters $\beta$ and $\varpi$, in Eqs. (4), we solved particular cases of the BGK and S-model to compare with Refs. [26] and [27], with which we found good agreement. Then, in order to establish a general and complete comparison, we generate results, showed in Tables 1 to 18 , for a set of parameters to which we had available in the literature results provided by the ADO method, for other kinetic models than the GJ and MRS models. In particular, results provided by the CES model $[29,30]$ and also the LBE equation [38]. It is important to remark that differently of the models defined in Eqs. (4), the CES model components [10] have to be evaluated by solving some integral equations, and the difficulties and the computational effort involved in its implementation are much more significant. Same observation is valid for the results obtained from the LBE equation [38], where, in fact, the ADO method is associated with the coefficients of a proposed expansion of the solution in terms of Legendre functions. We believe these comments are relevant, in respect to the analysis we try to establish in this work.

And so, Tables 1 to 4 are related to the different quantities of interest evaluated for the Poiseuille flow problem. Results for the thermal-creep problem are listed in Tables 5 to 7 . In Tables 8 to 12, the results showed are associated with the Couette flow problem. An analysis of the thermal-slip and viscous-slip problems may be based on Tables 13 to 15 and Tables 16 to 18 , respectively.

Here we note that, we used the Onsager's relation [39]-[44], $U_{T}=Q_{P}$, as an additional way of checking our results for the Poiseuille and thermal-creep problem, and so, we supressed the related repeated results in Tables 5 to 7 . Another variation of the Onsager's relation that yields a useful relationship between the thermal-slip coefficient and the heat flow of the viscous-slip problem [44, 45] was also checked. We follow Siewert [45] and write this relation as

$$
\frac{1}{k_{0}} \int_{0}^{\infty} q_{k}(\tau) \mathrm{d} \tau=\frac{\varepsilon_{p} \varepsilon_{t}}{\varepsilon_{a}^{2}}\left[\gamma-\left(\varepsilon_{b} / \varepsilon_{t}\right) \frac{A_{s}}{k_{T}}\right]
$$

with

$$
\gamma=\frac{8}{15 \pi^{1 / 2} \varepsilon_{p} \varepsilon_{t}} \int_{0}^{\infty} e^{-c^{2}} A(c) B(c) c^{3} \mathrm{~d} c
$$


and where, $\varepsilon_{a}$ assumes the given value for $\varepsilon$, in Eq. (18), when the viscous-slip problem is solved. Analogously, $\varepsilon_{b}$ assumes the given value for $\varepsilon$, in Eq. (18), when the thermal-slip problem is solved. Still, in Eq. (115), the correspondent approximations for the Chapman-Ensgok functions [19, 10], $A(c)$ and $B(c)$, related to all four kinetic models we treat in this work, are expressed as $[10,34,11]$

$$
A(c)=\varepsilon_{t} c\left(c^{2}-5 / 2\right), \quad B(c)=\varepsilon_{p} c^{2} .
$$

If we substitute Eqs. (116) into Eq. (115), we find that $\gamma=1 / 2$, for all four kinetic models (BGK, S, GJ and MRS). This result agrees with previous result by Siewert [45] obtained for the BGK and the S model. In this way, we rewrite Eq. (114) as

$$
\frac{1}{k_{0}} \int_{0}^{\infty} q_{k}(\tau) \mathrm{d} \tau=\frac{\varepsilon_{p} \varepsilon_{t}}{\varepsilon_{a}^{2}}\left[1 / 2-\left(\varepsilon_{b} / \varepsilon_{t}\right) \frac{A_{s}}{k_{T}}\right]
$$

which was used to check our results, according Eqs. (108) and (106), for $k_{0}=k_{T}=$ 1. In fact, from these equations we can still write

$$
\sum_{j=1}^{2 N-1} A_{j} \nu_{j} \mathbf{Y}\left(\nu_{j}\right)=\frac{\varepsilon_{p} \varepsilon_{t}}{\varepsilon_{a}}\left[1 / 2-\left(\varepsilon_{b} / \varepsilon_{t}\right) A_{s}\right] .
$$

In particular, for different choices of $\varepsilon_{a}$ and $\varepsilon_{b}$, we were able to confirm the results given in Table 15, for the BGK, S, GJ and MRS models.

Next, in Tables 19 to 26, we chose the GJ model to generate some results for channel problems defined by surfaces with different accommodation coefficients, since it has been of interest these days [46], and, at the same time, not many results are available in the literature.

An analysis of the general class of cases we have studied lead us to point out some concluding comments:

- Considering the three kinetic models which gives a correct value for Prandtl number $\left(\varepsilon_{p} / \varepsilon_{t}\right.$ ratio), the S-model, the GJ model and the MRS model, which solution here are developed from the same main procedure, a detailed view [47] seems to point out that, the MRS model shows results in better agreement (in general 2 to 3 digits) with the CES and the LBE results; except for the Poiseuille flow and thermal-creep problems, where the GJ model works better, in this sense.

- In most of the cases the CES model results show better agreement, in general one more digit, than other models, when compared with the LBE results. However we call the attention to the fact that, the ADO method applied to this formulation is not included in the derivation presented here, and more significant analytical and computational efforts have to be considered.

- When the BGK model is included in the comparison, a better agreement between the dimensionless results provided for all the models depends, of course, on the choice of the mean-free-path.

A much more detailed analysis was taken into account [47], however, it would be too long to present here. But, surely, we can say that having a fast solution was very helpful in the generation of all the results [47]. 


\section{Concluding remarks}

A complete derivation of the solution of a class of kinetic equations - BGK, S, GJ and MRS models - based on an analytical version of the discrete-ordinates method, the ADO method, was presented. Several problems of the rarefied gas dynamics were solved based on this approach. A general comparison and analysis of those results with results provided by the LBE equation was established. The fact of using the same method, with analytical character, has been an important aspect and motivation when a comparative analysis of accurate results has to be carried out. Considering the analytical and computational effort versus the results, it seems that the GJ and MRS models add some improvements with respect to the other constant collision frequency models. In addition, it is important to emphasize that the ADO solution can be seen as a quite general procedure, in the sense of being able to deal with all this wide class of problems under the same basic procedures. In subsequent papers, we show that, except for minor changes in regard to the components of the matrix given by Eqs. (21) and the exact solutions expressed in Eqs. (79) and (80), the ADO solution is also valid in this general sense to problems associated with heat-transfer and evaporation processes.

\section{Acknowledgement}

This work is partially supported by CNPq - Conselho Nacional de Desenvolvimento Científico e Tecnológico do Brasil.

\section{References}

[1] C. Cercignani, The Boltzmann Equation and Its Applications, Springer-Verlag, New York 1988.

[2] Th. Bock and K. Jousten, Experimental verification of a Monte-Carlo simulation of the gas density in a vacuum chamber, Vacuum, 81 (2006), 234.

[3] J. Fan, I. D. Boyd, C. P. Cai, K. Hennighausen and G. V. Candler, Computation of rarefied gas flows around a NACA 0012 airfoil, AIAA, 39 (2001), 318.

[4] G. E. Karniadakis and A. Beskok, Micro Flows, Springer-Verlag, New York 2002.

[5] C. Cercignani, Rarefied Gas Dynamics From Basic Concepts to Actual Calculations, Cambridge Univ. Press, Cambridge 2000.

[6] R. Benzi, S. Succi and M. Vergassola, The lattice Boltzmann equation: theory and applications, Phys. Rev., 222 (1992), 145.

[7] P. L. Bhatnagar, E. P. Gross and M. Krook, A model for collision processes in gases. I. Small amplitude processes in charged and neutral one-component systems, Phys. Rev., 94 (1954), 511.

[8] E. P. Gross and E. A. Jackson, Kinetic models and the linearized Boltzmann equation, The Phys. of Fluids, 2 (1959), 432.

[9] E. M. Shakhov, Generalization of the Krook kinetic relaxation equation, Fluid Dynamics, 3 (1968), 142. (In Russian) 
[10] L. B. Barichello and C. E. Siewert, Some comments on modeling the linearized Boltzmann equation, J. Quant. Spect. Radiat. Transfer, 77 (2003), 43.

[11] R. D. M. Garcia and C. E. Siewert, The linearized Boltzmann equation: sound-wave propagation in a rarefied gas, Z. Angew. Math. Phys., 57 (2006), 94.

[12] R. D. M. Garcia and C. E. Siewert, The McCormack model for gas mixtures: heat transfer in a plane channel, Phys. Fluids, 16 (2004), 3393.

[13] R. F. Knackfuss and L. B. Barichello, On the temperature-jump problem in rarefied gas dynamics: the effect of the Cercignani-Lampis boundary condition, SIAM J. Appl. Math., 66 (2006), 2149.

[14] F. J. McCormack, Construction of linearized kinetic models for gaseous mixtures and molecular gases, Phys. Fluids, 16 (1973), 2095.

[15] C. Cercignani, The method of elementary solutions for kinetic models with velocitydependent collision frequency, Annals of Phys., 40 (1966), 469.

[16] S. K. Loyalka and J. H. Ferziger, Model dependence of the temperature slip coefficient, Phys. Fluids, 11 (1968), 1668.

[17] C. Cercignani, Mathematical Methods in Kinetic Theory, 2nd ed., Plenum Press, New York 1990.

[18] T. C. Cheng and S. K. Loyalka, Sound wave propagation in a rarefied gas - II: Gross-Jackson model, Progress in Nuclear Energy, 8 (1981), 263.

[19] S. K. Loyalka and K. A. Hickey, Plane Poiseuille flow: near continuum results for a rigid sphere gas, Physica A, 160 (1989), 395.

[20] S. Taguchi, K. Aoki and S. Takata, Vapor flows in the continuum limit in the presence of a small amount of noncondensable gas, Phys. Fluids, 16 (2004), 4105.

[21] M. M. R. Williams, A review of the rarefied gas dynamics theory associated with some classical problems in flow and heat transfer, Z. Angew. Math. Phys., 52 (2001), 500.

[22] Y. Sone, Kinetic theory and fluid dynamics, Birkhäuser, Boston 2002.

[23] F. Sharipov and V. Seleznev, Data on internal rarefied gas flows, J. Phys. Chem. Ref. Data, 27 (1998), 657.

[24] D. Valougeorgis, An analytical solution of the S-model kinetic equations, Z. Angew. Math. Phys., 54 (2003), 112.

[25] L. B. Barichello and C. E. Siewert, A discrete-ordinates solution for a non-grey model with complete frequency redistribution, J. Quant. Spect. Radiat. Transfer, 62 (1999), 665.

[26] L. B. Barichello, M. Camargo, P. Rodrigues and C. E. Siewert, Unified solutions to classical flow problems based on the BGK model, Z. Angew. Math. Phys., 52 (2001), 1.

[27] L. C. Cabrera and L. B. Barichello, Unified solutions to some classical problems in rarefied gas dynamics based on the one-dimensional linearized S-model equations, Z. Angew. Math. Phys., 57 (2006), 285.

[28] M. Camargo and L. B. Barichello, Unified approach for variable collision frequency models in rarefied gas dynamics, Transp. Theory Stat. Phys., 33 (2004), 227.

[29] C. E. Siewert, Poiseuille, thermal creep and Couette flow: results based on the CES model of the linearized Boltzmann equation, Euro. J. Mech. B/Fluids, 21 (2002), 579.

[30] C. E. Siewert, Two half-space problems based on a synthetic-kernel model of the linearized Boltzmann equation, J. Quant. Spect. Radiat. Transfer, 75 (2002), 21.

[31] C. L. Pekeris and Z. Alterman, Solution of the Boltzmann-Hilbert integral equation II. The coefficients of viscosity and heat conduction, Proc. Natl. Acad. Science, 43 (1957), 998.

[32] C. E. Siewert, Generalized boundary conditions for the S-model kinetic equations basic to flow in a plane channel, J. Quant. Spect. Radiat. Transfer, 72 (2002), 75.

[33] C. E. Siewert and F. Sharipov, Model equations in rarefied gas dynamics: viscous-slip and thermal-slip coefficients, Phys. Fluids, 14 (2002), 4123

[34] C. S. Scherer, Kinetics models of the linearized Boltzmann equation and a heat transfer problem in microscale (In Portuguese), MSc. Dissertation, Graduate Program in Applied Mathematics, Universidade Federal do Rio Grande do Sul, Porto Alegre, RS, Brasil (2005). 
[35] R. F. Knackfuss and L. B. Barichello, Surface effects in rarefied gas dynamics: an analysis based on the Cercignani-Lampis boundary condition, Euro. J. Mechanics B Fluids, 25 (2006), 113.

[36] J. J. Dongarra, J. R. Bunch, C. B. Moler and G. W. Stewart, LINPACK user's guide, SIAM, Philadelphia 1979

[37] B. T. Smith, J. M. Boyle, J. J. Dongarra, B. S. Garbow, Y. Ikebe, V. C. Klema, C. B. Moler, Matrix eigensystem routines - EISPACK guide, Springer-Verlag, Berlin 1976.

[38] C. E. Siewert, The linearized Boltzmann equation: concise and accurate solutions to basic flow problems, Z. Angew. Math. Phys., 54 (2003), 273.

[39] L. Onsager, Reciprocal relations in irreversible processes. I, Phys. Rev., 37 (1931), 405.

[40] L. Onsager, Reciprocal relations in irreversible processes. II, Phys. Rev., 38 (1931), 2265.

[41] F. Sharipov, Onsager-Casimir reciprocity relations for open gaseous systems at arbitrary rarefaction. I. General theory for single gas, Physica A, 203 (1994), 437.

[42] F. Sharipov, Onsager-Casimir reciprocity relations for open gaseous systems at arbitrary rarefaction. II. Application of the theory for single gas, Physica A, 203 (1994), 457.

[43] F. Sharipov, Onsager-Casimir reciprocity relations for open gaseous systems at arbitrary rarefaction. III. Theory and its application for gaseous mixtures, Physica A, 209 (1994), 457.

[44] F. Sharipov, Onsager-Casimir reciprocal relations based on the Boltzmann equation and gas-surface interaction: single gas, Phys. Rev. E, 73 (2006), 026110.

[45] C. E. Siewert, On computing the thermal-slip coefficient from Kramer's problem, Phys. Fluids, 16 (2004), 2132.

[46] C. Cercignani, M. Lampis and S. Lorenzani, Variational approach to gas flows in microchannels, Phys. Fluids, 16 (2004), 3426.

[47] J. F. Prolo Filho, The Boltzmann equation modeling flows in micro and macro scales (In Portuguese), MSc. Dissertation, Graduate Program in Applied Mathematics, Universidade Federal do Rio Grande do Sul, Porto Alegre, RS, Brasil (2007). 


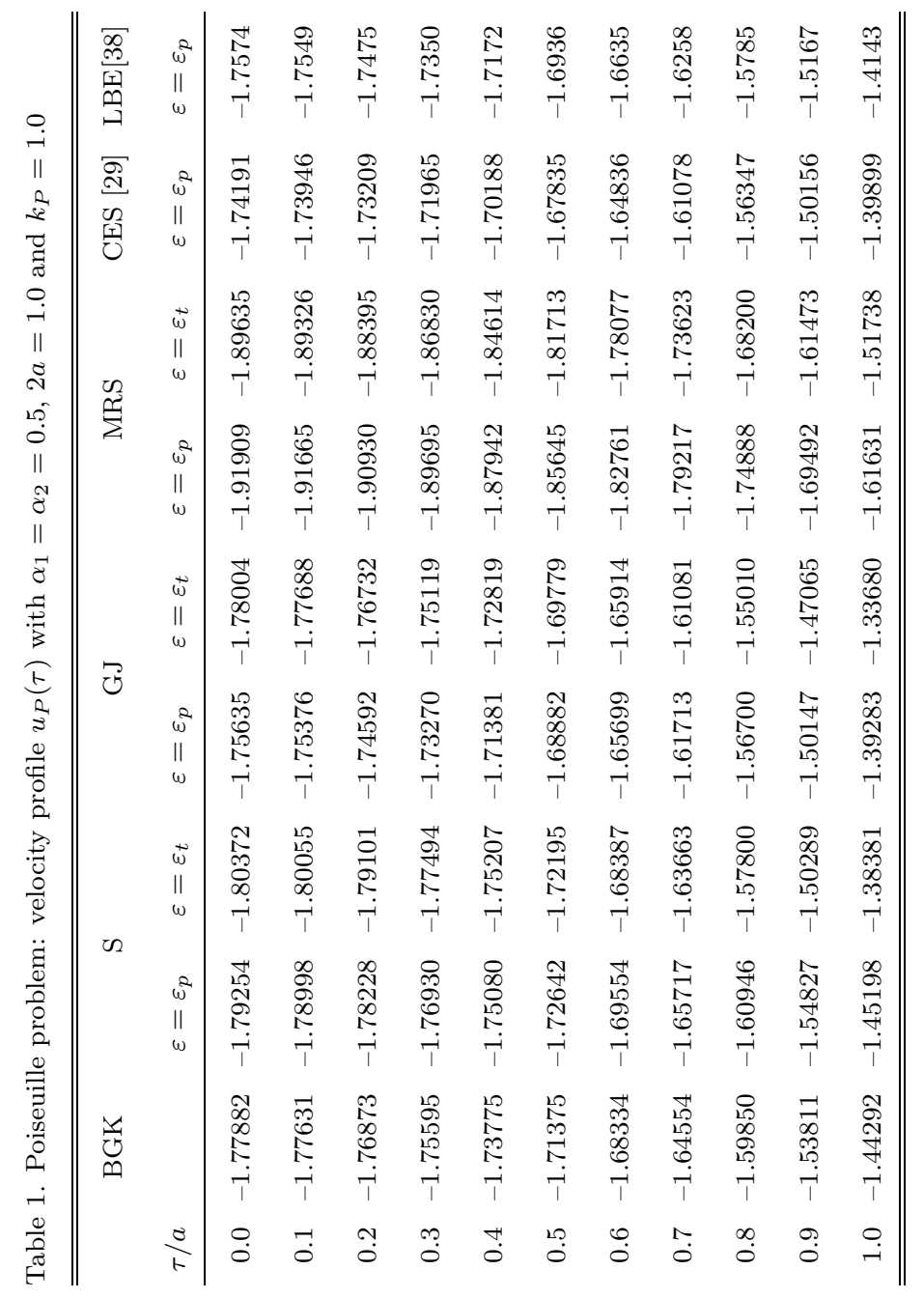




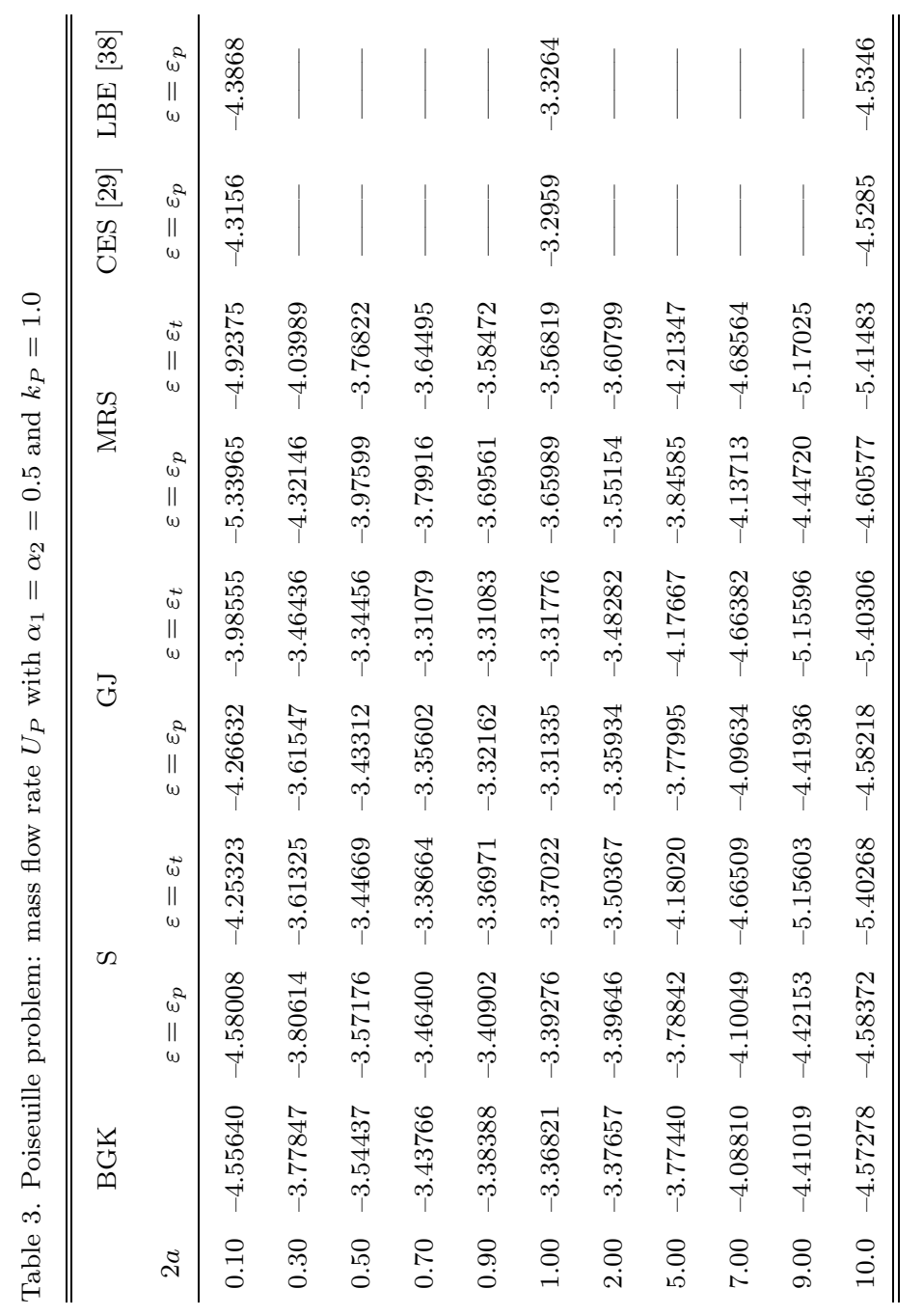




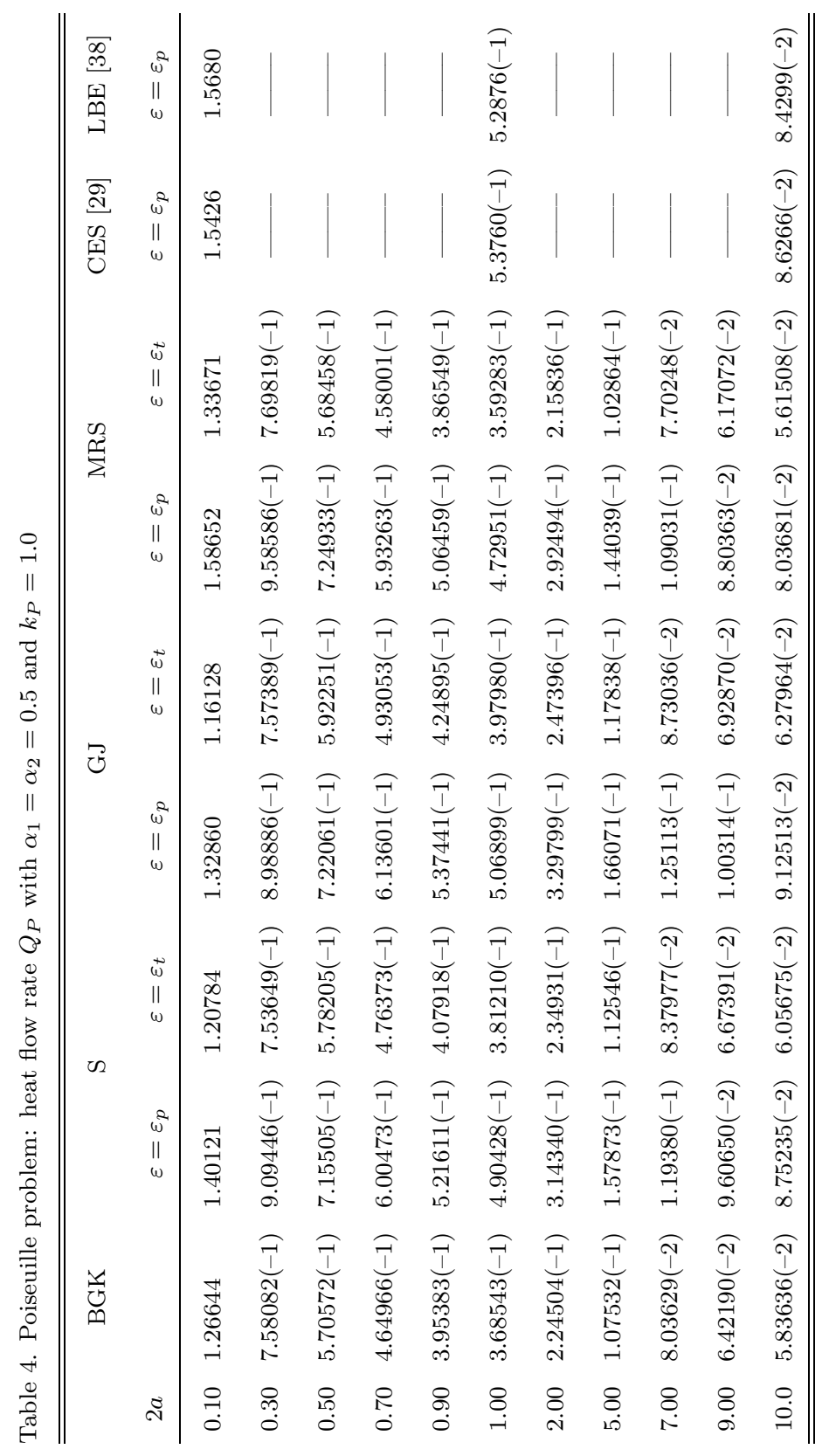




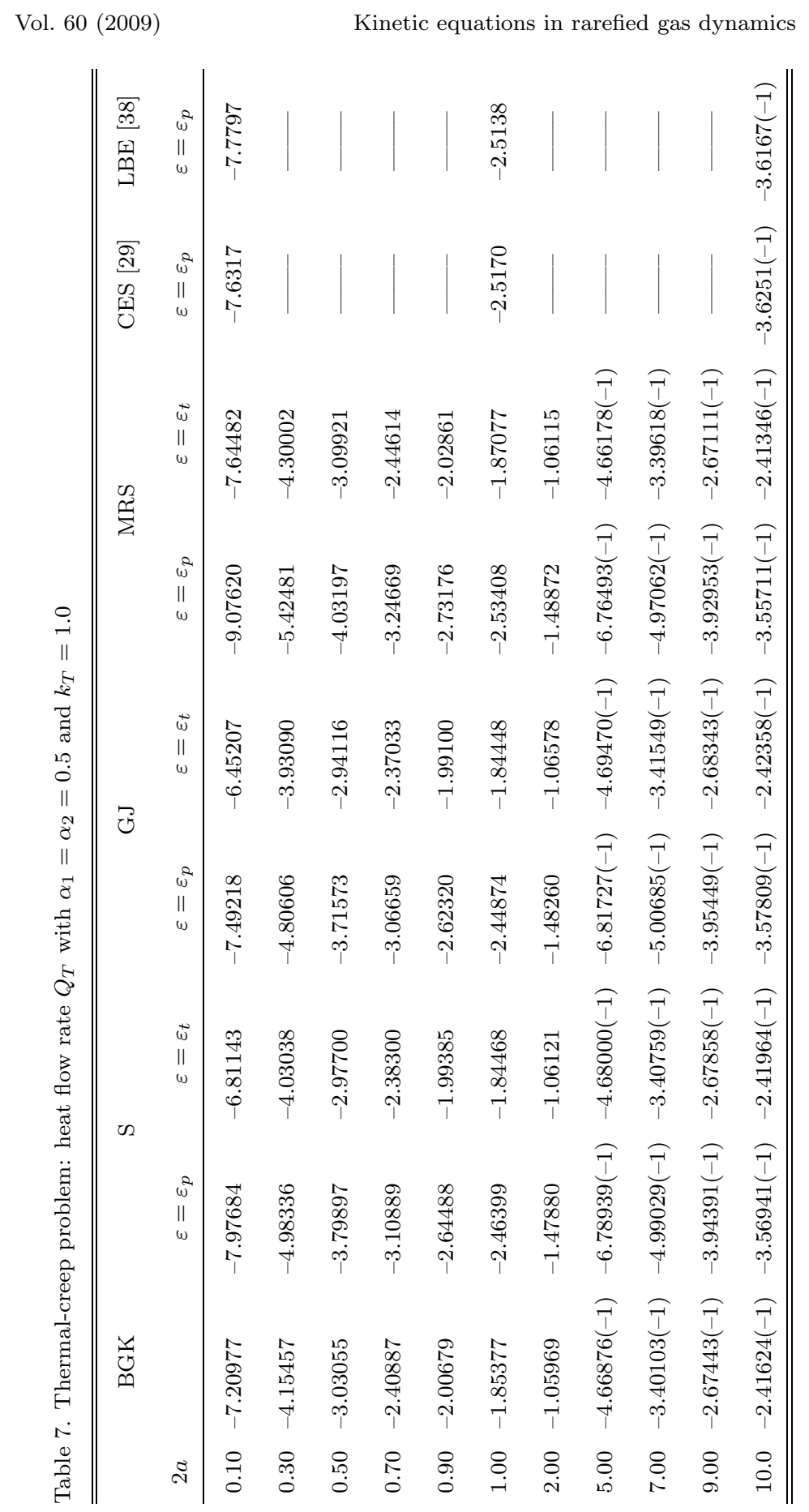




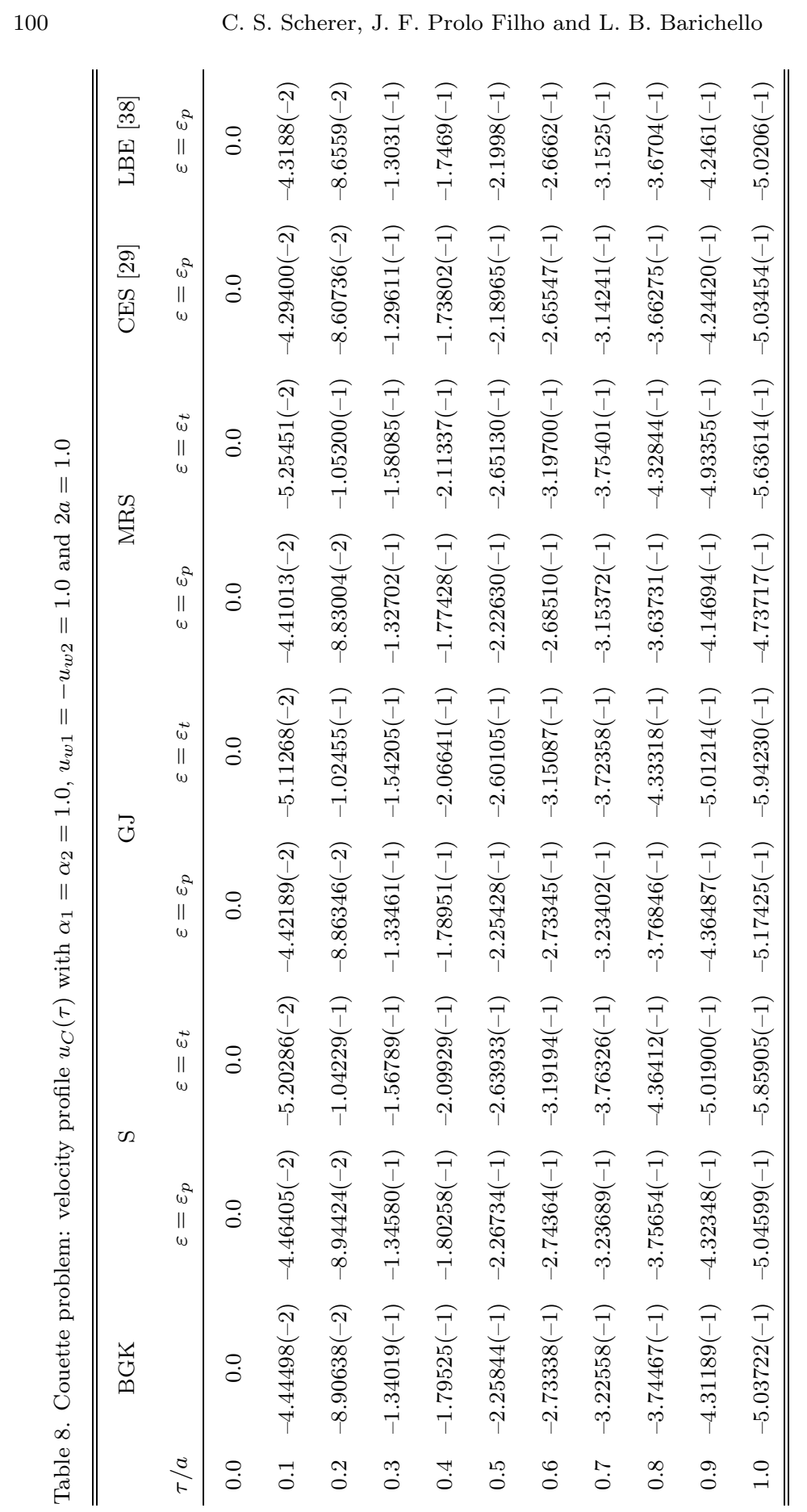




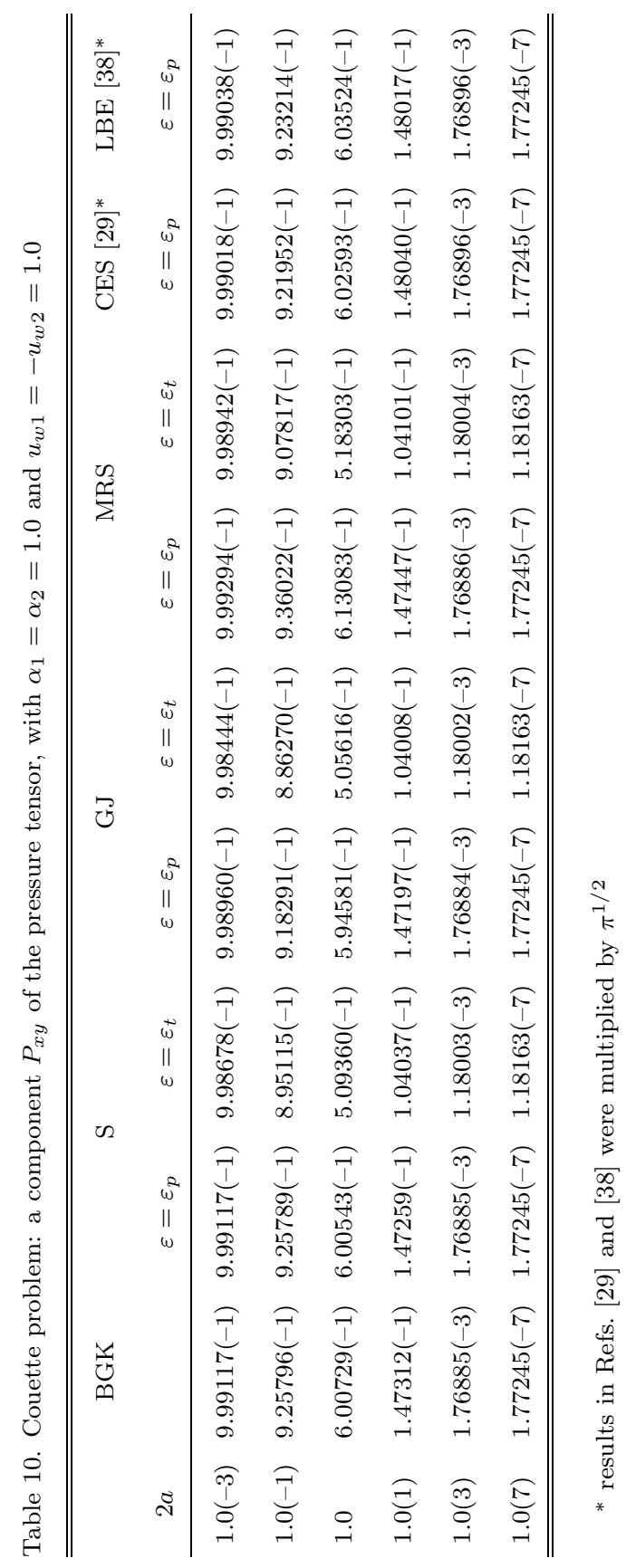




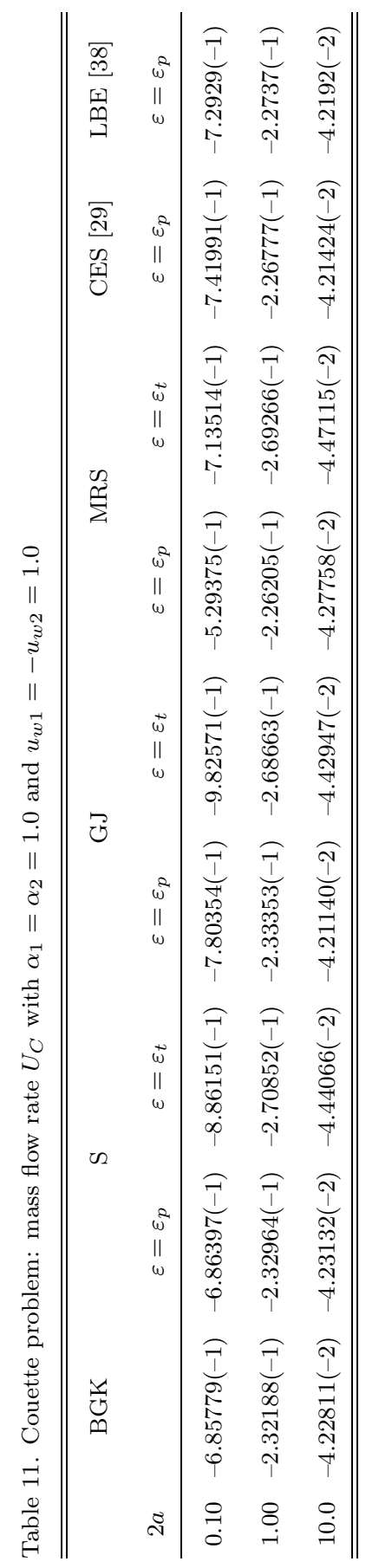




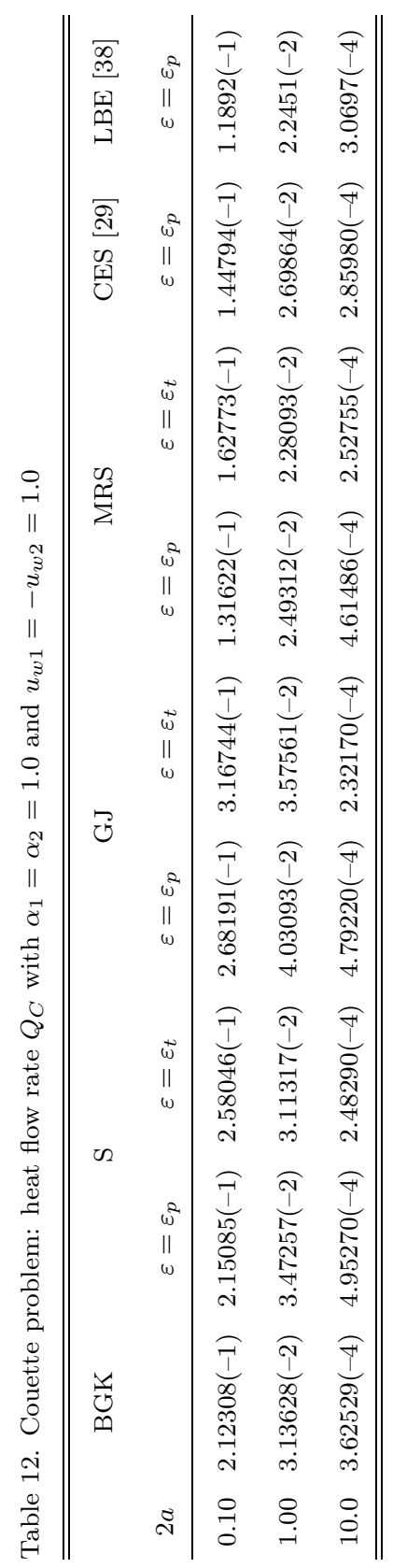




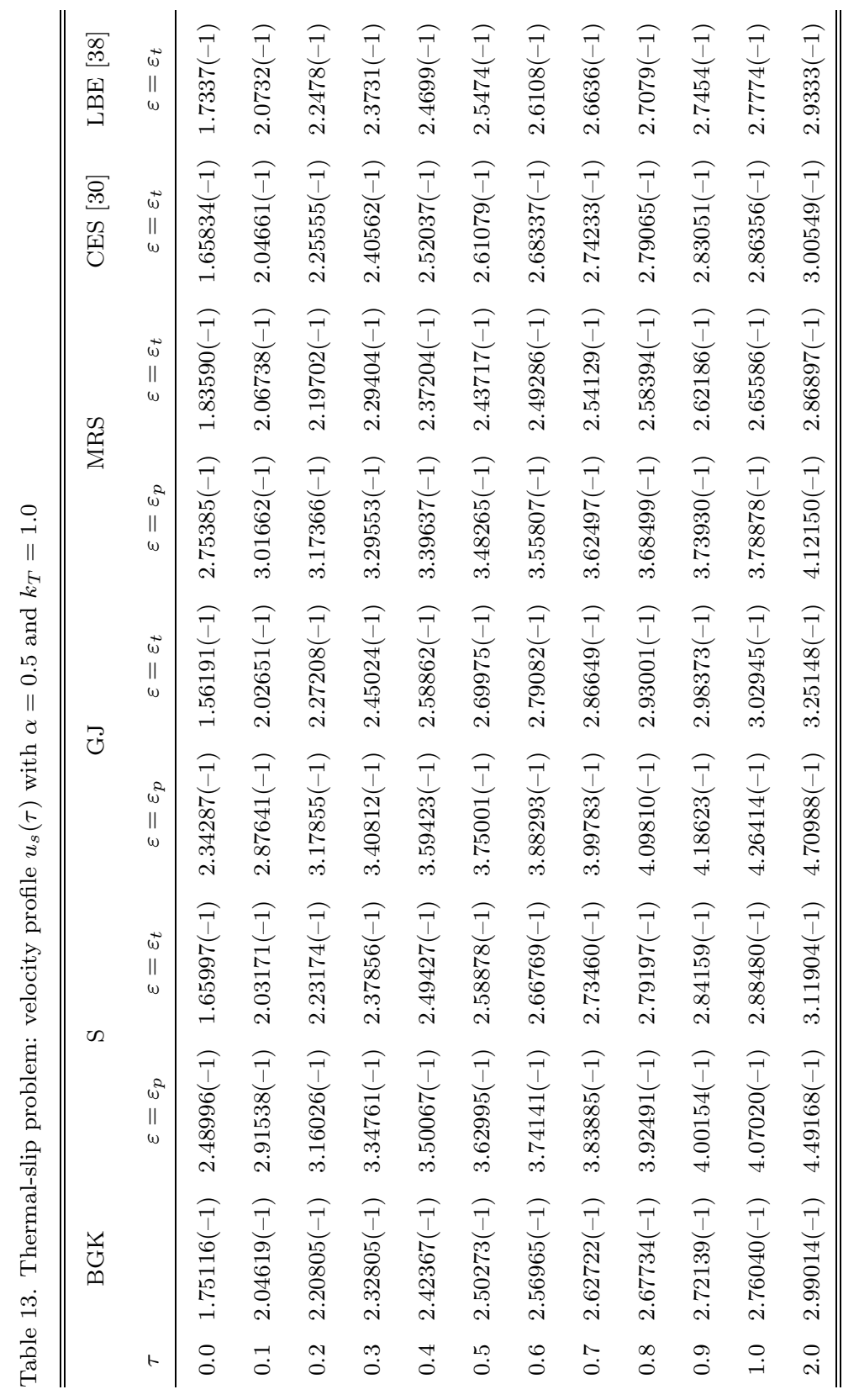




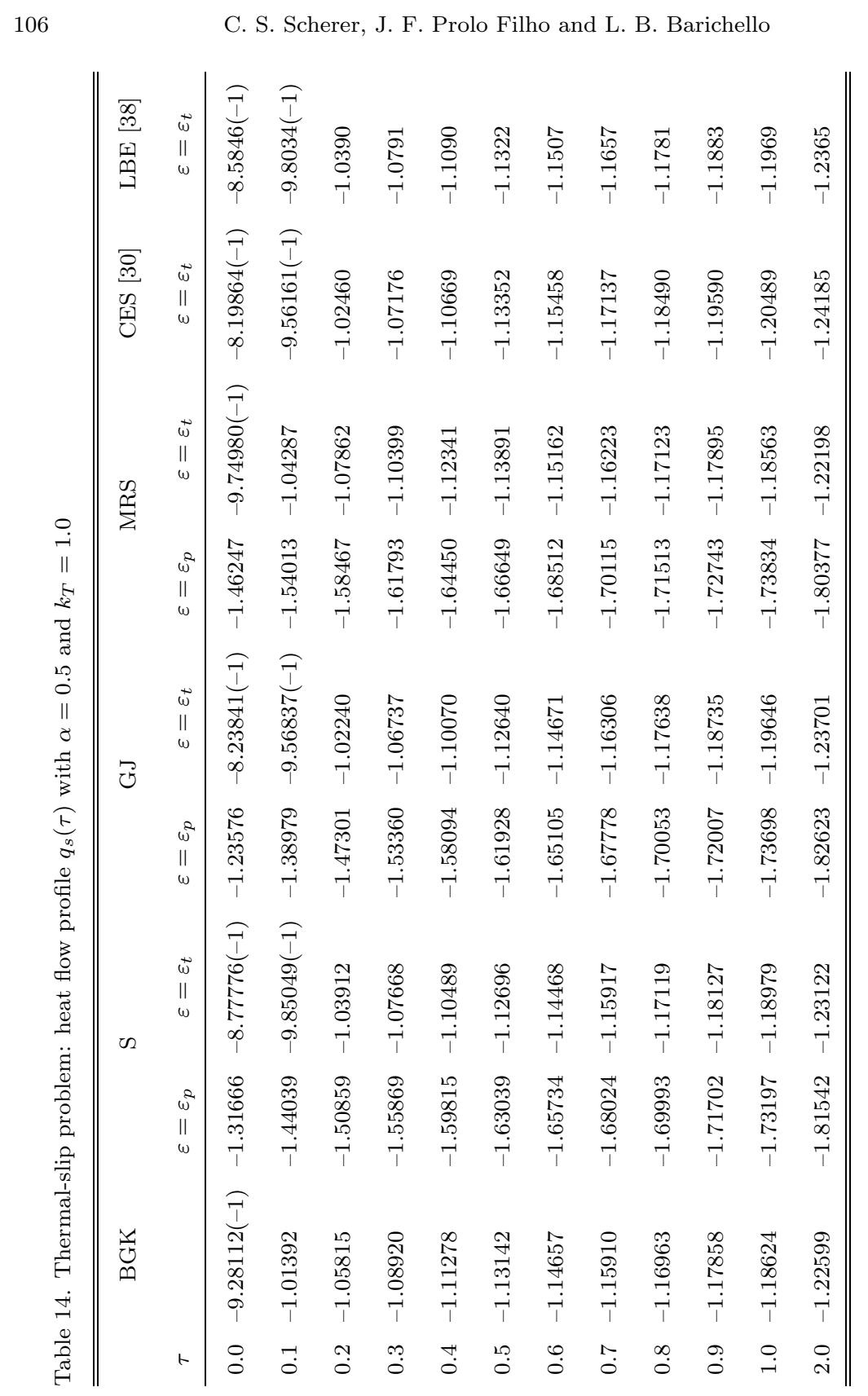




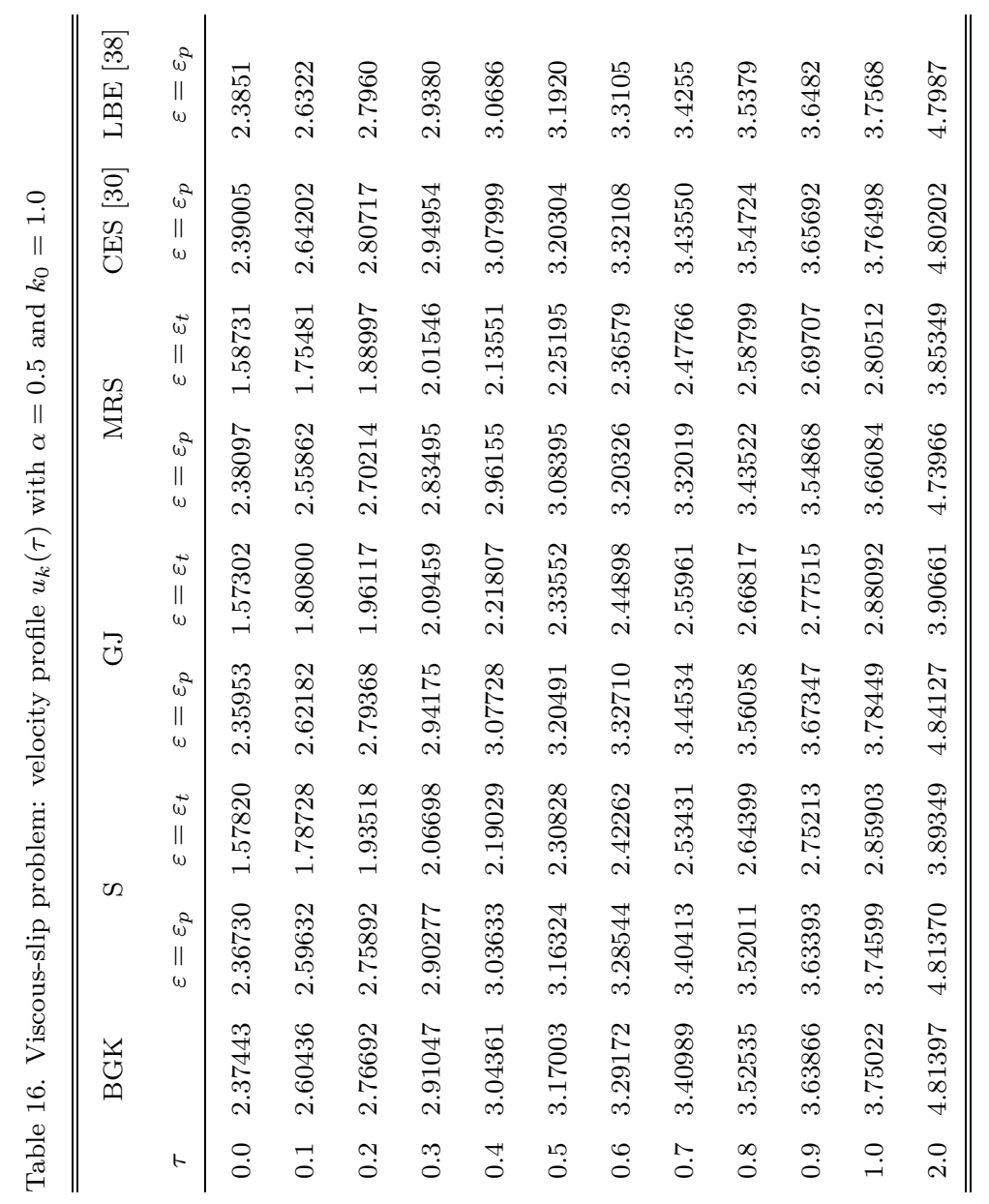




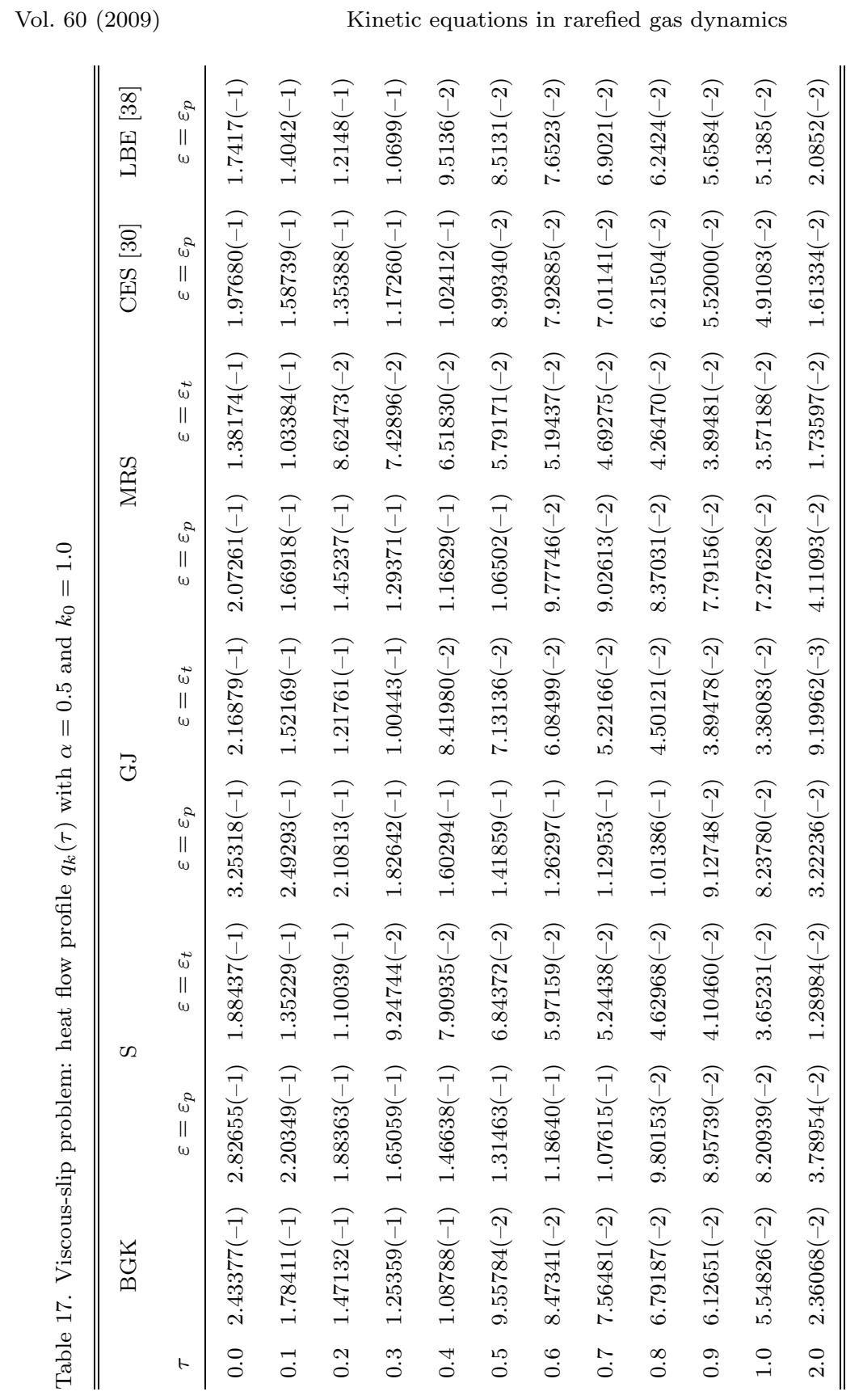


Table 19. Poiseuille problem: mass flow rate $U_{P}$ with GJ model, $\varepsilon=\varepsilon_{p}$ and $k_{P}=1.0$

\begin{tabular}{cccccc}
\hline \hline & $\alpha_{1}=0.5$ & $\alpha_{1}=0.5$ & $\alpha_{1}=0.5$ & $\alpha_{1}=0.5$ & $\alpha_{1}=0.5$ \\
$2 a$ & $\alpha_{2}=0.1$ & $\alpha_{2}=0.3$ & $\alpha_{2}=0.5$ & $\alpha_{2}=0.8$ & $\alpha_{2}=1.0$ \\
\hline 0.10 & -6.70315 & -5.29293 & -4.26632 & -3.15486 & -2.59659 \\
0.30 & -5.85677 & -4.52999 & -3.61547 & -2.67425 & -2.22107 \\
0.50 & -5.66560 & -4.32458 & -3.43312 & -2.54315 & -2.12461 \\
0.70 & -5.61234 & -4.24121 & -3.35602 & -2.49242 & -2.09302 \\
0.90 & -5.61241 & -4.20648 & -3.32162 & -2.47465 & -2.08808 \\
1.00 & -5.62314 & -4.19914 & -3.31335 & -2.47268 & -2.09121 \\
2.00 & -5.87222 & -4.26638 & -3.35934 & -2.55324 & -2.20256 \\
5.00 & -6.83729 & -4.75063 & -3.77995 & -3.01116 & -2.69895 \\
7.00 & -7.45341 & -5.09721 & -4.09634 & -3.34049 & -3.04174 \\
9.00 & -8.03572 & -5.44352 & -4.41936 & -3.67276 & -3.38338 \\
10.0 & -8.31538 & -5.61607 & -4.58218 & -3.83930 & -3.55363 \\
\hline \hline
\end{tabular}

Table 20. Poiseuille problem: mass flow rate $U_{P}$ with GJ model, $\varepsilon=\varepsilon_{p}$ and $k_{P}=1.0$

\begin{tabular}{cccccc}
\hline \hline & $\alpha_{1}=1.0$ & $\alpha_{1}=1.0$ & $\alpha_{1}=1.0$ & $\alpha_{1}=1.0$ & $\alpha_{1}=1.0$ \\
$2 a$ & $\alpha_{2}=0.1$ & $\alpha_{2}=0.3$ & $\alpha_{2}=0.5$ & $\alpha_{2}=0.8$ & $\alpha_{2}=1.0$ \\
\hline 0.10 & -3.23646 & -2.90617 & -2.59659 & -2.16688 & -1.90112 \\
0.30 & -2.85427 & -2.51716 & -2.22107 & -1.83855 & -1.61688 \\
0.50 & -2.79553 & -2.43057 & -2.12461 & -1.74792 & -1.53852 \\
0.70 & -2.80942 & -2.41283 & -2.09302 & -1.71399 & -1.50984 \\
0.90 & -2.85252 & -2.42289 & -2.08808 & -1.70385 & -1.50222 \\
1.00 & -2.88011 & -2.43365 & -2.09121 & -1.70388 & -1.50295 \\
2.00 & -3.23995 & -2.61963 & -2.20256 & -1.78063 & -1.57939 \\
5.00 & -4.43491 & -3.29187 & -2.69895 & -2.19815 & -1.98647 \\
7.00 & -5.17873 & -3.71708 & -3.04174 & -2.50829 & -2.29176 \\
9.00 & -5.87113 & -4.12225 & -3.38338 & -2.82660 & -2.60667 \\
10.0 & -6.19999 & -4.31897 & -3.55363 & -2.98757 & -2.76631 \\
\hline \hline
\end{tabular}


Table 21. Poiseuille problem: heat flow rate $Q_{P}$ with GJ model, $\varepsilon=\varepsilon_{p}$ and $k_{P}=1.0$

\begin{tabular}{llllll}
\hline \hline & $\alpha_{1}=0.5$ & $\alpha_{1}=0.5$ & $\alpha_{1}=0.5$ & $\alpha_{1}=0.5$ & $\alpha_{1}=0.5$ \\
$2 a$ & $\alpha_{2}=0.1$ & $\alpha_{2}=0.3$ & $\alpha_{2}=0.5$ & $\alpha_{2}=0.8$ & $\alpha_{2}=1.0$ \\
\hline 0.10 & 1.89586 & 1.57432 & 1.32860 & 1.05026 & $9.05271(-1)$ \\
0.30 & 1.20945 & 1.03139 & $8.98886(-1)$ & $7.51688(-1)$ & $6.76059(-1)$ \\
0.50 & $9.31226(-1)$ & $8.09273(-1)$ & $7.22061(-1)$ & $6.27882(-1)$ & $5.80308(-1)$ \\
0.70 & $7.66249(-1)$ & $6.75364(-1)$ & $6.13601(-1)$ & $5.49593(-1)$ & $5.18165(-1)$ \\
0.90 & $6.54228(-1)$ & $5.83048(-1)$ & $5.37441(-1)$ & $4.92662(-1)$ & $4.71600(-1)$ \\
1.00 & $6.10346(-1)$ & $5.46517(-1)$ & $5.06899(-1)$ & $4.69228(-1)$ & $4.52005(-1)$ \\
2.00 & $3.69403(-1)$ & $3.41520(-1)$ & $3.29799(-1)$ & $3.24064(-1)$ & $3.23770(-1)$ \\
5.00 & $1.70712(-1)$ & $1.64802(-1)$ & $1.66071(-1)$ & $1.71603(-1)$ & $1.75984(-1)$ \\
7.00 & $1.25460(-1)$ & $1.22854(-1)$ & $1.25113(-1)$ & $1.30534(-1)$ & $1.34412(-1)$ \\
9.00 & $9.90403(-2)$ & $9.79012(-2)$ & $1.00314(-1)$ & $1.05183(-1)$ & $1.08513(-1)$ \\
10.0 & $8.95692(-2)$ & $8.88622(-2)$ & $9.12513(-2)$ & $9.58381(-2)$ & $9.89302(-2)$ \\
\hline \hline
\end{tabular}

Table 22. Poiseuille problem: heat flow rate $Q_{P}$ with GJ model, $\varepsilon=\varepsilon_{p}$ and $k_{P}=1.0$

\begin{tabular}{cccccc}
\hline \hline & $\alpha_{1}=1.0$ & $\alpha_{1}=1.0$ & $\alpha_{1}=1.0$ & $\alpha_{1}=1.0$ & $\alpha_{1}=1.0$ \\
$2 a$ & $\alpha_{2}=0.1$ & $\alpha_{2}=0.3$ & $\alpha_{2}=0.5$ & $\alpha_{2}=0.8$ & $\alpha_{2}=1.0$ \\
\hline 0.10 & 1.11449 & 1.00512 & $9.05271(-1)$ & $7.71244(-1)$ & $6.91176(-1)$ \\
0.30 & $8.42671(-1)$ & $7.52178(-1)$ & $6.76059(-1)$ & $5.83048(-1)$ & $5.32260(-1)$ \\
0.50 & $7.24337(-1)$ & $6.44134(-1)$ & $5.80308(-1)$ & $5.06887(-1)$ & $4.68986(-1)$ \\
0.70 & $6.44485(-1)$ & $5.72715(-1)$ & $5.18165(-1)$ & $4.58380(-1)$ & $4.28865(-1)$ \\
0.90 & $5.83055(-1)$ & $5.18600(-1)$ & $4.71600(-1)$ & $4.22264(-1)$ & $3.98865(-1)$ \\
1.00 & $5.56864(-1)$ & $4.95717(-1)$ & $4.52005(-1)$ & $4.07053(-1)$ & $3.86145(-1)$ \\
2.00 & $3.83564(-1)$ & $3.45979(-1)$ & $3.23770(-1)$ & $3.05262(-1)$ & $2.98443(-1)$ \\
5.00 & $1.92524(-1)$ & $1.79718(-1)$ & $1.75984(-1)$ & $1.76217(-1)$ & $1.77937(-1)$ \\
7.00 & $1.43006(-1)$ & $1.35332(-1)$ & $1.34412(-1)$ & $1.36643(-1)$ & $1.38944(-1)$ \\
9.00 & $1.13331(-1)$ & $1.08295(-1)$ & $1.08513(-1)$ & $1.11264(-1)$ & $1.13559(-1)$ \\
10.0 & $1.02582(-1)$ & $9.84083(-2)$ & $9.89302(-2)$ & $1.01742(-1)$ & $1.03971(-1)$ \\
\hline \hline
\end{tabular}


Table 23. Thermal-creep problem: heat flow rate $Q_{T}$ with GJ model, $\varepsilon=\varepsilon_{p}$ and $k_{T}=1.0$

\begin{tabular}{cccccc}
\hline \hline & $\alpha_{1}=0.5$ & $\alpha_{1}=0.5$ & $\alpha_{1}=0.5$ & $\alpha_{1}=0.5$ & $\alpha_{1}=0.5$ \\
$2 a$ & $\alpha_{2}=0.1$ & $\alpha_{2}=0.3$ & $\alpha_{2}=0.5$ & $\alpha_{2}=0.8$ & $\alpha_{2}=1.0$ \\
\hline 0.10 & $-1.05048(1)$ & -8.84162 & -7.49218 & -5.87342 & -4.98851 \\
0.30 & -6.26190 & -5.47381 & -4.80606 & -3.97230 & -3.50205 \\
0.50 & -4.62535 & -4.13891 & -3.71573 & -3.17335 & -2.86080 \\
0.70 & -3.69524 & -3.36174 & -3.06659 & -2.68148 & -2.45613 \\
0.90 & -3.08434 & -2.84104 & -2.62320 & -2.33537 & -2.16507 \\
1.00 & -2.85011 & -2.63878 & -2.44874 & -2.19642 & -2.04646 \\
2.00 & -1.62388 & -1.55018 & -1.48260 & -1.39081 & -1.33508 \\
5.00 & $-7.08241(-1)$ & $-6.94452(-1)$ & $-6.81727(-1)$ & $-6.64309(-1)$ & $-6.53660(-1)$ \\
7.00 & $-5.14364(-1)$ & $-5.07252(-1)$ & $-5.00685(-1)$ & $-4.91698(-1)$ & $-4.86204(-1)$ \\
9.00 & $-4.03745(-1)$ & $-3.99432(-1)$ & $-3.95449(-1)$ & $-3.89998(-1)$ & $-3.86668(-1)$ \\
10.0 & $-3.64533(-1)$ & $-3.61037(-1)$ & $-3.57809(-1)$ & $-3.53393(-1)$ & $-3.50695(-1)$ \\
\hline \hline
\end{tabular}

Table 24. Thermal-creep problem: heat flow rate $Q_{T}$ with GJ model, $\varepsilon=\varepsilon_{p}$ and $k_{T}=1.0$

\begin{tabular}{cccccc}
\hline \hline & $\alpha_{1}=1.0$ & $\alpha_{1}=1.0$ & $\alpha_{1}=1.0$ & $\alpha_{1}=1.0$ & $\alpha_{1}=1.0$ \\
$2 a$ & $\alpha_{2}=0.1$ & $\alpha_{2}=0.3$ & $\alpha_{2}=0.5$ & $\alpha_{2}=0.8$ & $\alpha_{2}=1.0$ \\
\hline 0.10 & -6.01184 & -5.49157 & -4.98851 & -4.26400 & -3.79974 \\
0.30 & -4.16422 & -3.82332 & -3.50205 & -3.05262 & -2.77228 \\
0.50 & -3.35743 & -3.10019 & -2.86080 & -2.53032 & -2.32658 \\
0.70 & -2.84648 & -2.64361 & -2.45613 & -2.19914 & -2.04163 \\
0.90 & -2.47999 & -2.31598 & -2.16507 & -1.95897 & -1.83305 \\
1.00 & -2.33135 & -2.18289 & -2.04646 & -1.86039 & -1.74680 \\
2.00 & -1.45893 & -1.39425 & -1.33508 & -1.25455 & -1.20546 \\
5.00 & $-6.79836(-1)$ & $-6.66183(-1)$ & $-6.53660(-1)$ & $-6.36560(-1)$ & $-6.26110(-1)$ \\
7.00 & $-4.99830(-1)$ & $-4.92728(-1)$ & $-4.86204(-1)$ & $-4.77289(-1)$ & $-4.71841(-1)$ \\
9.00 & $-3.94950(-1)$ & $-3.90635(-1)$ & $-3.86668(-1)$ & $-3.81245(-1)$ & $-3.77933(-1)$ \\
10.0 & $-3.57409(-1)$ & $-3.53912(-1)$ & $-3.50695(-1)$ & $-3.46298(-1)$ & $-3.43612(-1)$ \\
\hline \hline
\end{tabular}


Table 25. Couette problem: component $P_{x y}$ of the pressure tensor, GJ model, $\varepsilon=\varepsilon_{p}$ and $u_{w 1}=-u_{w 2}=1.0$

\begin{tabular}{lccccc}
\hline \hline & $\alpha_{1}=0.5$ & $\alpha_{1}=0.5$ & $\alpha_{1}=0.5$ & $\alpha_{1}=0.5$ & $\alpha_{1}=0.5$ \\
\multicolumn{1}{c}{$2 a$} & $\alpha_{1}=0.1$ & $\alpha_{2}=0.3$ & $\alpha_{2}=0.5$ & $\alpha_{2}=0.8$ & $\alpha_{2}=1.0$ \\
\hline $1.0(-1)$ & $9.01195(-2)$ & $2.25802(-1)$ & $3.23169(-1)$ & $4.26788(-1)$ & $4.77939(-1)$ \\
1.0 & $8.50593(-2)$ & $1.96912(-1)$ & $2.67784(-1)$ & $3.36756(-1)$ & $3.68962(-1)$ \\
$1.0(1)$ & $5.90856(-2)$ & $9.76856(-2)$ & $1.12564(-1)$ & $1.23364(-1)$ & $1.27572(-1)$ \\
$1.0(3)$ & $1.73770(-3)$ & $1.75813(-3)$ & $1.76232(-3)$ & $1.76474(-3)$ & $1.76557(-3)$ \\
$1.0(7)$ & $1.77245(-7)$ & $1.77245(-7)$ & $1.77245(-7)$ & $1.77245(-7)$ & $1.77245(-7)$ \\
\hline \hline
\end{tabular}

Table 26. Couette problem: component $P_{x y}$ of the pressure tensor, GJ model, $\varepsilon=\varepsilon_{p}$ and $u_{w 1}=-u_{w 2}=1.0$

\begin{tabular}{lccccc}
\hline \hline & $\alpha_{1}=1.0$ & $\alpha_{1}=1.0$ & $\alpha_{1}=1.0$ & $\alpha_{1}=1.0$ & $\alpha_{1}=1.0$ \\
\multicolumn{1}{c}{$2 a$} & $\alpha_{2}=0.1$ & $\alpha_{2}=0.3$ & $\alpha_{2}=0.5$ & $\alpha_{2}=0.8$ & $\alpha_{2}=1.0$ \\
\hline $1.0(-1)$ & $9.90573(-2)$ & $2.91796(-1)$ & $4.77939(-1)$ & $7.46110(-1)$ & $9.18291(-1)$ \\
1.0 & $9.31454(-2)$ & $2.46543(-1)$ & $3.68962(-1)$ & $5.14717(-1)$ & $5.94581(-1)$ \\
$1.0(1)$ & $6.29742(-2)$ & $1.08792(-1)$ & $1.27572(-1)$ & $1.41624(-1)$ & $1.47197(-1)$ \\
$1.0(3)$ & $1.74086(-3)$ & $1.76137(-3)$ & $1.76557(-3)$ & $1.76800(-3)$ & $1.76884(-3)$ \\
$1.0(7)$ & $1.77245(-7)$ & $1.77245(-7)$ & $1.77245(-7)$ & $1.77245(-7)$ & $1.77245(-7)$ \\
\hline \hline
\end{tabular}




\section{S. Scherer}

Programa de Pós-Graduação em Engenharia Mecânica

Universidade Federal do Rio Grande do Sul

Rua Sarmento Leite, 425

90050-170 Porto Alegre, RS

Brasil

e-mail: csscherer@unisinos.br

Permanent address:

Universidade do Vale do Rio dos Sinos

Unisinos, São Leopoldo, RS

Brasil

J. F. Prolo Filho

Programa de Pós-Graduação em Matemática Aplicada e Computacional e-mail: joprolo@gmail.com

L. B. Barichello

Instituto de Matemática

Universidade Federal do Rio Grande do Sul

Av. Bento Gonçalves, 9500 - Campus do Vale

91509-900 Porto Alegre, RS

Brasil

e-mail: lbaric@ufrgs.br

(Received: July 10, 2007; revised: October 29/December 4, 2007)

Published Online First: July 5, 2008

To access this journal online: www.birkhauser.ch/zamp 